\title{
The Nitsche XFEM-DG Space-Time Method and its Implementation in Three Space Dimensions
}

\author{
Christoph Lehrenfeld *
}

\author{
Bericht Nr. 377
}

Oktober 2013

Key words: finite elements, evolving surface, parabolic PDE, space-time, two-phase flow, computations, XFEM, $4 \mathrm{D}$ quadrature, discontinuous galerkin

AMS Subject Classifications: 65D30, 65M30

Institut für Geometrie und Praktische Mathematik RWTH Aachen

Templergraben 55, D-52056 Aachen (Germany)

* Institut für Geometrie und Praktische Mathematik, RWTH Aachen, D-52056 Aachen, Germany; email: lehrenfeld@igpm.rwth-aachen.de 


\title{
THE NITSCHE XFEM-DG SPACE-TIME METHOD AND ITS IMPLEMENTATION IN THREE SPACE DIMENSIONS
}

\author{
CHRISTOPH LEHRENFELD *
}

\begin{abstract}
We consider a standard model for mass transport across an evolving interface. The solution has to satisfy a jump condition across an evolving interface. In the recent paper [C. Lehrenfeld, A. Reusken, SIAM J. Num. Anal., 51 (2013)] a new finite element discretization method for this mass transport problem is presented and analyzed. This method is based on a space-time approach in which a discontinuous Galerkin (DG) technique (in time) is combined with an extended finite element method (XFEM). The jump condition is satisfied in a weak sense by using the Nitsche method. While the emphasis in that paper was on the analysis and one dimensional numerical experiments the main contribution of this paper is the discussion of implementation aspects for the spatially three dimensional case. As the space-time interface is typically given only implicitly as the zero-level of a level-set function, we construct a piecewise planar approximation of the space-time interface. This discrete interface is used to divide the space-time domain into its subdomains. An important component within this decomposition is a new method for dividing four-dimensional prisms intersected by a piecewise planar space-time interface into simplices. Such a subdivision algorithm is necessary for numerical integration on the subdomains as well as on the space-time interface. These numerical integrations are needed in the implementation of the Nitsche XFEM-DG method in three space dimensions. Corresponding numerical studies are presented and discussed.
\end{abstract}

Key words. finite elements, evolving surface, parabolic PDE, space-time, two-phase flow, computations, XFEM, 4D quadrature, discontinuous galerkin

AMS subject classifications. 65D30, 65M 30

1. Introduction. Let $\Omega \subset \mathbb{R}^{d}, d=2,3$, be a convex polygonal domain that contains two different immiscible incompressible phases. The (in general time dependent) subdomains containing the two phases are denoted by $\Omega_{1}, \Omega_{2}$, with $\bar{\Omega}=\bar{\Omega}_{1} \cup \bar{\Omega}_{2}$. For simplicity we assume that $\partial \Omega_{1} \cap \partial \Omega=\emptyset$, i.e. the phase in $\Omega_{1}$ is completely surrounded by the one in $\Omega_{2}$. The interface $\Gamma:=\bar{\Omega}_{1} \cap \bar{\Omega}_{2}$ is assumed to be sufficiently smooth. A model example is a (rising) droplet in a flow field. The fluid dynamics in such a flow problem is often modeled by the incompressible Navier-Stokes equations combined with suitable conditions at the interface which describe the effect of surface tension. For this model we refer to the literature, e.g. [3, 8, 16, 9]. By w we denote the velocity field resulting from these Navier-Stokes equations. We assume that $\operatorname{div} \mathbf{w}=0$ holds. Furthermore, we assume that the evolution of the interface is determined by this velocity field, in the sense that $V_{\Gamma}=\mathbf{w} \cdot \mathbf{n}$ holds, where $V_{\Gamma}$ is the normal velocity of the interface and $\mathbf{n}$ denotes the unit normal at $\Gamma$ pointing from $\Omega_{1}$ into $\Omega_{2}$. We consider a standard model which describes the transport of a dissolved species $u$ in a two-phase flow problem. In strong formulation this model is as follows:

$$
\begin{aligned}
\frac{\partial u}{\partial t}+\mathbf{w} \cdot \nabla u-\operatorname{div}(\alpha \nabla u) & =f \quad \text { in } \Omega_{m}(t), m=1,2, t \in[0, T], \\
{[\alpha \nabla u \cdot \mathbf{n}]_{\Gamma} } & =0, \\
{[\beta u]_{\Gamma} } & =0, \\
u(\cdot, 0) & =u_{0} \text { in } \Omega_{m}(0), m=1,2, \\
u(\cdot, t) & =0 \text { on } \partial \Omega, \quad t \in[0, T] .
\end{aligned}
$$

*Institut für Geometrie und Praktische Mathematik, RWTH Aachen, D-52056 Aachen, Germany; email: lehrenfeld@igpm.rwth-aachen.de 
For a sufficiently smooth function $v,[v]=[v]_{\Gamma}$ denotes the jump of $v$ across $\Gamma$, i.e. $[v]=\left(v_{1}\right)_{\mid \Gamma}-\left(v_{2}\right)_{\mid \Gamma}$, where $v_{m}=v_{\mid \Omega_{m}}$ is the restriction of $v$ to $\Omega_{m}$. In (1.1) we have standard parabolic convection-diffusion equations in the two subdomains $\Omega_{1}$ and $\Omega_{2}$. In many applications one has a homogeneous problem, i.e. $f \equiv 0$. The diffusion coefficient $\alpha=\alpha(\mathbf{x}, t)$ is assumed to be piecewise constant:

$$
\alpha=\alpha_{m}(t)>0 \text { in } \Omega_{m}(t) .
$$

In general we have $\alpha_{1} \neq \alpha_{2}$. The interface condition in (1.2) results from the conservation of mass principle. The condition in (1.3) is the so-called Henry condition, cf. $[23,22,4,3]$. In this condition the coefficient $\beta=\beta(\mathbf{x}, t)$ is strictly positive and piecewise constant:

$$
\beta=\beta_{m}(t)>0 \text { in } \Omega_{m}(t) .
$$

In general we have $\beta_{1} \neq \beta_{2}$, since species concentration usually has a jump discontinuity at the interface due to different solubilities within the respective fluid phases. Hence, the solution $u$ is discontinuous across the (evolving) interface. Both for the mathematical analysis and numerical treatment of this transport problem there is a big difference between the case with a stationary interface and the one with a non-stationary interface. In this paper we discuss several aspects of the finite element discretization method proposed in [20] for a non-stationary interface problem.

In recent years it has been shown that for such a transport problem with an (evolving) interface the Nitsche-XFEM method is very well suited [10, 21]. In [11, 12, 13, 1, 5] the application of the Nitsche-XFEM to other classes of problems is studied. In [10] this method is analyzed for a stationary heat diffusion problem (no convection) with a conductivity that is discontinuous across the interface $\left(\alpha_{1} \neq \alpha_{2}\right)$ but with a solution that is continuous across the interface $\left(\beta_{1}=\beta_{2}\right)$. In $[21,19]$ the method is studied for the parabolic problem described above, with $\beta_{1} \neq \beta_{2}$ (discontinuous solution), and with a (dominating) convection term in (1.1). In all these papers, and in other literature that we know of, the Nitsche XFEM method is analyzed for a two-phase transport as in (1.1)-(1.5) with a stationary interface.

In [20] an analysis for the case of a non-stationary interface is presented. In that paper the restriction to the diffusion dominated case is made, i.e., no stabilization w.r.t. convection is needed.

For the weak formulation of the parabolic mass transport problem (1.1)-(1.5) with a non-stationary interface a space-time variational formulation is most natural, cf. chapter 10 in [9]. This suggests an XFEM approach combined with a suitable spacetime discontinuous Galerkin method. The main result in [20] is a full (i.e. in space and time) discretization that has (proven) second order accuracy (in space and time) for a transport problem as (1.1)-(1.5), with a solution that is discontinuous across an evolving interface. The space-time XFEM-DG method is an Eulerian method in the sense that the spatial triangulation is not fitted to the interface. The idea of combining space-time and extended finite elements has already been presented in [6] and applied to spatially one dimensional conservation equations without any analysis.

In implementations of the spatially three dimensional case one has to deal with four dimensional geometries. In the literature space-time discretizations which explicitly construct four dimensional triangulations are rare. In [2] a decomposition of four dimensional prisms into simplices is applied to achieve real space-time adaptivity within one time slab. More global four dimensional simplex triangulations are considered in $[18,17]$. 
The main new contributions of this paper are the following two:

1. A strategy to decompose the space-time domain which is intersected by an implicitly defined space-time interface into simplices and

2. numerical studies for the Nitsche XFEM-DG method in three space dimension. structure and content of this paper. In section 2 we present the discretization method that is considered in the analysis in [20]. This method is often not feasible in practice, due to the fact that it is assumed that volume integrals close to the space-time interface and surface integrals over the space-time interface are evaluated exactly. In practice a strategy for approximating these integrals will be necessary. Implementation aspects are discussed in section 3. Especially a strategy to construct a piecewise planar space-time interface approximation and corresponding polygonal approximations of the space-time subdomains is presented. A crucial point in this strategy is the decomposition of prisms (intersected by a piecewise planar interface) into (uncut) simplices. In the spatially three dimensional case this involves four dimensional geometries. A main contribution of this paper is a solution algorithm for this problem that is presented separately in section 5 . In section 4 numerical experiments in three spatial dimensions are presented and discussed.

2. The Nitsche XFEM-DG discretization. In this section we present the discretization method. This method will have the form of a variational problem in a certain space-time finite element space. The space-time domain is denoted by $Q=\Omega \times(0, T] \subset \mathbb{R}^{d+1}$. A partitioning of the time interval is given by $0=t_{0}<$ $t_{1}<\ldots<t_{N}=T$. By considering the time slabs $Q^{n}=\Omega \times I_{n}$ with $I_{n}:=\left(t_{n-1}, t_{n}\right]$ one after another, the computational complexity is comparable to other (implicit) time-stepping schemes. In the following we will consider the problem on one time slab $Q^{n}$, we will therefore omit the superscript $n$.

Corresponding to the time interval $I_{n}:=\left(t_{n-1}, t_{n}\right]$ we assume a given shape regular simplicial triangulation $\mathcal{T}$ of the spatial domain $\Omega$. In general this triangulation is not fitted to the interface $\Gamma(t)$. Note, that for different time slabs this triangulation $\mathcal{T}$ is allowed to change (cf. figure 2.1). Corresponding to the triangulation of $\Omega$ into $d$-dimensional simplices $\mathcal{T}=\{T\}$, we have a canonical triangulation of $Q$ into $d+1$-dimensional prisms. This triangulation is denoted by $\mathcal{T}^{*}=\left\{\mathcal{Q}^{T}\right\}$ where for each prism we have $\mathcal{Q}^{T}=T \times I_{n}$ for a corresponding $d$-dimensional simplex $T$.

In the discretization the same space is used for both trial and test functions. We introduce the method for the case of piecewise bilinear space-time functions (linear in space and linear in time). Let $V_{h}$ be the finite element space of continuous piecewise linear functions on $\mathcal{T}$ with zero boundary values on $\partial \Omega$. The spatial mesh size parameter corresponding to $V_{h}$ is denoted by $h$. Corresponding space-time finite element spaces on the time slab $Q$ are given by

$$
W:=\left\{v: Q \rightarrow \mathbb{R} \mid v(\mathbf{x}, t)=\phi_{0}(\mathbf{x})+t \phi_{1}(\mathbf{x}), \quad \phi_{0}, \phi_{1} \in V_{h}\right\}
$$

In the time slab $Q$ we define the subdomains $Q_{m}:=\bigcup_{t \in I_{n}} \Omega_{m}(t), m=1,2$. We introduce corresponding canonical restrictions $R_{m}$ on $L^{2}(Q)$ given by $R_{m} v=\left.v\right|_{Q_{m}}$. We will also use the notation $v_{m}:=R_{m} v$. The space-time XFEM spaces are given by

$$
W^{\Gamma_{*}}:=R_{1} W \oplus R_{2} W
$$

The symbol $\Gamma_{*}$ denotes the space-time interface in $Q$, i.e., $\Gamma_{*}:=\cup_{t \in I_{n}} \Gamma(t)$. Note that $\Gamma_{*}$ depends on the time interval $I_{n}$. 


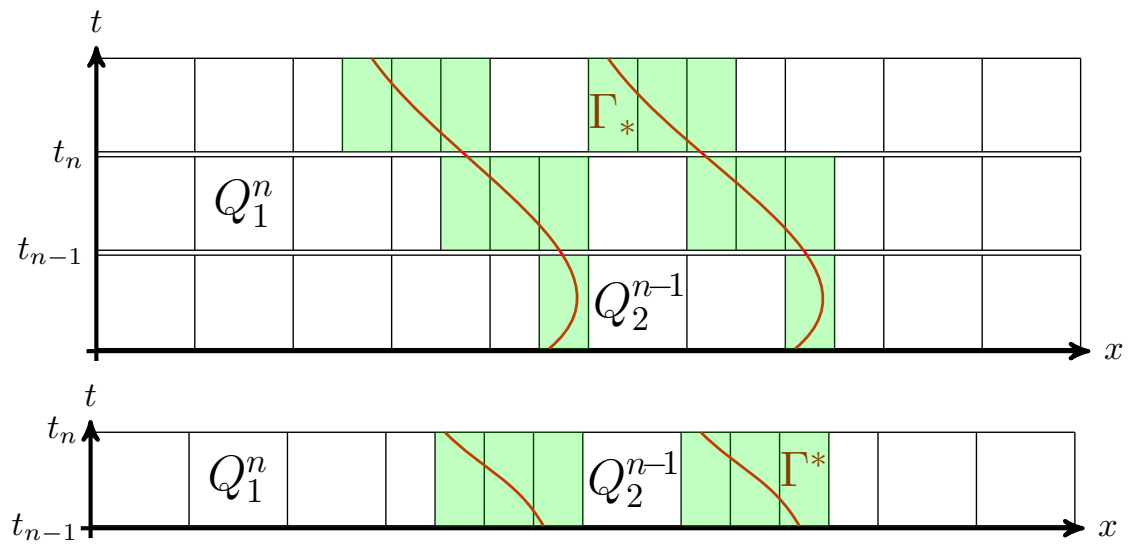

FIG. 2.1. Sketch of the space-time domains $Q_{m}^{n}$. Note that within each time slab the triangulation has a tensor product structure $\mathcal{T} \times\left[t_{n-1}, t_{n}\right]$. The triangulation is allowed to change between the time slabs.

We will treat the Henry condition $[\beta u]_{\Gamma}=0$ using the Nitsche technique (in space-time). For this we need suitable averages and jumps across $\Gamma_{*}$, that we now introduce. Take $t \in I_{n}, T \in \mathcal{T}$ and let $\mathcal{Q}_{m}^{T}:=\mathcal{Q}^{T} \cap Q_{m}$. We define the weights

$$
\kappa_{m \mid \mathcal{Q}^{T}}:=\frac{\left|\mathcal{Q}_{m}^{T}\right|}{\left|\mathcal{Q}^{T}\right|}
$$

and the corresponding averaging operator on the space-time interface

$$
\{v\}_{\Gamma_{*}}(\mathbf{x}, t):=\kappa_{1}\left(v_{1}\right)_{\mid \Gamma_{*}}+\kappa_{2}\left(v_{2}\right)_{\mid \Gamma_{*}}, \quad(\mathbf{x}, t) \in \Gamma_{*} .
$$

REMARK 2.1. The choice for $\left.\kappa_{m}\right|_{\mathcal{Q}^{T}}$ is slightly different from the one in [20]. As $\left.\kappa_{m}\right|_{\mathcal{Q}^{T}}$ is time-independent within one element $\mathcal{Q}^{T}$ it is better suited for implementation. One can easily check that the theoretical results in [20] carry over with only minor adjustments.

We use a similar notation for the jump operators:

$$
[v]_{\Gamma_{*}}(\mathbf{x}, t)=\left(v_{2}\right)_{\mid \Gamma_{*}}-\left(v_{1}\right)_{\mid \Gamma_{*}}, \quad(\mathbf{x}, t) \in \Gamma_{*} .
$$

In the discontinuous Galerkin method we need jump terms across the end points of the time intervals $I_{n}=\left(t_{n-1}, t_{n}\right]$. We define $u_{+}^{n-1}(\cdot):=\lim _{\epsilon \downarrow 0} u\left(\cdot, t_{n-1}+\epsilon\right)$ and introduce the notation

$$
v^{n}(\mathbf{x}):=v\left(\mathbf{x}, t_{n}\right), \quad[v]^{n}(\mathbf{x}):=v_{+}^{n}(\mathbf{x})-v^{n}(\mathbf{x}), \quad 0 \leq n \leq N-1, \quad \text { with } \quad v^{0}(\mathbf{x}):=0 .
$$

On the cross sections $\Omega \times\left\{t_{n}\right\}, 0 \leq n \leq N$, of $Q$ we use a weighted $L^{2}$ scalar product

$$
(u, v)_{0, t_{n}}:=\int_{\Omega} \beta\left(\cdot, t_{n}\right) u v d \mathbf{x}=\sum_{m=1}^{2} \beta_{m} \int_{\Omega_{m}\left(t_{n}\right)} u v d \mathbf{x} .
$$

This scalar product is uniformly (w.r.t. $n$ and $N$ ) equivalent to the standard scalar product in $L^{2}(\Omega)$. Note that we use a weighting with $\beta$ in this scalar product, which is not reflected in the notation. 
In the Nitsche bilinear form introduced below we also use another weighted $L^{2}$ scalar product. Related to this we note that the surface measure both on $\Gamma(t) \subset \mathbb{R}^{d}$ and on $\Gamma_{*} \subset \mathbb{R}^{d+1}$ play a role. Both measures are denoted by $d \mathbf{s}$. The following transformation formula holds:

$$
\int_{0}^{T} \int_{\Gamma(t)} f(\mathbf{s}, t) d \mathbf{s} d t=\int_{\Gamma_{*}} f(\mathbf{s})\left(1+\left(\mathbf{w} \cdot \mathbf{n}_{\Gamma}\right)^{2}\right)^{-\frac{1}{2}} d \mathbf{s}=: \int_{\Gamma_{*}} f(\mathbf{s}) \nu(\mathbf{s}) d \mathbf{s}
$$

with $\nu(\mathbf{s})=\left(1+\left(\mathbf{w} \cdot \mathbf{n}_{\Gamma}\right)^{2}\right)^{-\frac{1}{2}}$ and $\mathbf{w}$ the (interface) velocity. We assume that the space-time interface is sufficiently smooth such that there is a constant $c_{0}>0$ with

$$
c_{0} \leq \nu(\mathbf{s}) \leq 1 \quad \text { for all } \mathbf{s} \in \Gamma_{*} .
$$

Below in the Nitsche bilinear form we use a weighting with $\nu$.

The notation introduced above is used to define a bilinear form $B(\cdot, \cdot)$, which consists of three parts, namely a term $a(\cdot, \cdot)$ that directly corresponds to the partial differential equation, a term $b(\cdot, \cdot)$ which weakly enforces continuity with respect to $t$ at the time interval end points $t_{n-1}$, and a term $N_{\Gamma_{*}}(\cdot, \cdot)$ which enforces in a weak sense the Henry condition $[\beta u]_{\Gamma_{*}}=0$. We now define the bi- and linear forms:

$$
\begin{aligned}
a(u, v)= & \sum_{m=1}^{2} \int_{Q_{m}}\left(\frac{\partial u_{m}}{\partial t}+\mathbf{w} \cdot \nabla u_{m}\right) \beta_{m} v_{m}+\alpha_{m} \beta_{m} \nabla u_{m} \cdot \nabla v_{m} d \mathbf{x} d t \\
N_{\Gamma_{*}}(u, v)= & -\int_{\Gamma_{*}} \nu\{\alpha \nabla u \cdot \mathbf{n}\}_{\Gamma_{*}}[\beta v]_{\Gamma_{*}} d \mathbf{s}-\int_{\Gamma_{*}} \nu\{\alpha \nabla v \cdot \mathbf{n}\}_{\Gamma_{*}}[\beta u]_{\Gamma_{*}} d \mathbf{s} \\
& +\lambda h_{T}^{-1} \int_{\Gamma_{*}} \nu[\beta u]_{\Gamma_{*}}[\beta v]_{\Gamma_{*}} d \mathbf{s}, \\
b(u, v)= & \left(u_{+}^{n-1}, v_{+}^{n-1}\right)_{0, t_{n-1}}, \\
f(v)= & \int_{Q^{n}} f \beta v d \mathbf{x} d t, \\
c(\bar{u} ; \cdot)= & \left(u^{n-1}, v_{+}^{n-1}\right)_{0, t_{n-1}} .
\end{aligned}
$$

where $\bar{u}$ should emphasize that $u^{n-1}$ is given (initial) data for the computations in time interval $I_{n}$, e.g. for $n=1, u^{n-1}$ is the initial data $u^{0}$ of $(1.4)$. In $N_{\Gamma_{*}}(\cdot, \cdot)$ the parameter $\lambda \geq 0$ has to be chosen sufficiently large. These bi- and linear forms are well-defined on the space-time XFEM space $W^{\Gamma_{*}}$ and the problem within one time slab reads as: Find $u \in W^{\Gamma_{*}}$, s.t.

$$
B(u, v):=a(u, v)+b(u, v)+N^{\Gamma_{*}}(u, v)=f(v)+c(\bar{u}, v) \quad \forall v \in W^{\Gamma_{*}}
$$

Note that the solution of (2.3) is the result of one time step within a time stepping scheme. In [20] it has been shown, that the presented method is a stable discretization scheme and a second order (in time and space) error bound for the $L^{2}(\Omega(T)$ )-norm has been proven.

3. Implementation aspects. In order to implement the method presented in section 2 one has to compute the matrices and vectors representing the bi- and linear forms. In section 3.1 we explain which types of integrals need to be calculated for that. In practice the space-time interface $\Gamma_{*}$ is typically not given explicitly, but implicitly, e.g. as the zero-level of a level-set function. In our application the level-set function 
is a piecewise quadratic function in space, and typically only given at discrete time levels $t^{n}$. In order to apply quadrature on the space-time objects, we want to have an explicit representation of the space-time interface. As this is practically not feasible, we construct an appropriate approximation $\Gamma_{*, h}$ which has an explicit representation. Such an approximation is discussed in section 3.2. A different approach is presented in the recent paper [15] where quadrature rules for implicitly given domains by means of moment-fitting are derived.

The special tensor product structure of each time slab is reflected in the construction and representation of finite element shape functions. A short discussion on the shape functions, especially w.r.t. XFEM can be found in section 3.3.

Once, the (space-time) geometries and finite element shape functions are defined one needs suitable quadrature rules for (regular) prisms and simplices. As these are discussed in many standard references (e.g. [24]) for the case $d+1 \leq 3$, but only rarely in the $3+1$-dimensional case, section 3.4 and section 3.5 address this issue. In section 3.4 tensor product quadrature rules for prisms are applied for elements that are not intersected. The more involved situation when elements are cut by $\Gamma_{*, h}$ make use of the decomposition of an intersected $d+1$-prism into $d+1$-simplices. This is discussed in 3.5 .

3.1. Integral types. Every bi- and linear form in (2.3) has a natural decomposition into its element contributions, e.g. $a(u, v)=\sum_{\mathcal{Q}^{T} \in \mathcal{T}^{*}} a^{T}(u, v)$. We consider the task of computing the element contributions of the (bi-)linear forms $a(\cdot, \cdot), b(\cdot, \cdot)$, $N_{\Gamma_{*}}(\cdot, \cdot), f(\cdot)$ and $c(\bar{u} ; \cdot)$.

As we need to calculate (approximations of) integrals of different kinds, we categorize these integrals before we discuss their numerical treatment. We distinguish those integrals in terms of the sets $S$ we are integrating on. The cases are denoted as case $(\mathrm{m}, \mathrm{n}, \mathrm{o})$ where $\mathrm{m}$ is the dimension of $S, \mathrm{n}$ is the co-dimension of $S$ and $\mathrm{o} \in\{\mathrm{c}, \mathrm{n}\}$ describes if the set $S$ is intersected by the space-time interface $\Gamma_{*}$ (o=c) or not (o=n). We recall the notation for a prism $\mathcal{Q}^{T}=T \times I_{n}$. Accordingly we define $\mathcal{Q}_{m}^{T}=\mathcal{Q}^{T} \cap Q_{m}$.

3.1.1. $a(\cdot, \cdot), f(\cdot): \quad d+1$-dimensional measure, co-dimension 0. Integrals appearing on each element for $a(\cdot, \cdot)$ are integrals on $d+1$-dimensional objects like

$$
\int_{\mathcal{Q}_{m}^{T}} f d \mathbf{x}=\int_{t_{n-1}}^{t_{n}} \int_{T_{m}(t)} f d \mathbf{x}, \text { with } T_{m}(t)=T \cap \Omega_{m}(t)
$$

We distinguish two different situations: The prism $\mathcal{Q}_{m}^{T}$ is not intersected by the (approximated) interface, i.e. the prism is completely in one phase and thus the volume to integrate on is the prism itself. We consider this as case $(d+1,0, n)$ where numerical integration can exploit the tensor product structure. If on the other hand the prism $\mathcal{Q}_{m}^{T}$ is intersected by the (approximated) interface, the geometry $\mathcal{Q}_{T}^{m}$ is much more difficult to handle. In that case $d+1$-dimensional quadrature on subsimplices has to be applied. This is denoted by case $(d+1,0, c)$.

3.1.2. $b(\cdot, \cdot), c(\hat{u}, \cdot)$ : $d$-dimensional measure, co-dimension 0 . The integrals in the element contributions of $b(\cdot, \cdot)$ and $c(\bar{u} ; \cdot)$ have the form

$$
\int_{T_{m}\left(t_{n-1}\right)} f d \mathbf{x}
$$

and thus are $d$-dimensional measures. Also here, we distinguish the case of a one phase element (i.e. an element which is not intersected), denoted by case $(\mathrm{d}, 0, \mathrm{n})$ and the case of an intersected element, case $(d, 0, c)$. 
3.1.3. $N^{\Gamma_{*}}(\cdot, \cdot)$ : $d$-dimensional measure, co-dimension 1. For the spacetime integrals stemming from the Nitsche stabilization bilinear form $N^{\Gamma_{*}}(\cdot, \cdot)$ on each element we get terms like

$$
\int_{\Gamma_{*}^{T}} \nu \cdot d \mathbf{s}
$$

where $\Gamma_{*}^{T}=\Gamma_{*} \cap \mathcal{Q}^{T}$. Some terms also depend on the normal direction $\mathbf{n}_{\Gamma}$. These integrals only appear on elements that are intersected. The measure is d-dimensional on the manifold $\Gamma_{*}$ with co-dimension 1 . This case is denoted as case $(\mathrm{d}, 1, \mathrm{c})$.

3.2. Approximation of the space-time interface $\Gamma_{*}$ and the space-time volumes $Q_{m}$. For the integration of space-time volumes and the space-time interface a discrete approximation of $\Gamma_{*}$ and $Q_{m}$ which is feasible for numerical integration has to be found. This is relevant for the integration cases $(d+1,0, c)$ and $(d, 1, c)$.

Note that case $(d, 0, c)$ is an integral on a d-dimensional simplex $T$. To deal with those we follow the strategies (for $d \leq 3$ ) discussed in [9, Section 7.3]. In order to get approximations $\Gamma_{*, h}$ and $Q_{m, h}$ to the space-time interface and volumes (case $(\mathrm{d}+1,0, \mathrm{c}))$ we proceed similarly as in lower $(d)$ dimensions.

We consider the prism $\mathcal{Q}^{T}$ with a characteristic spatial length of $T$ (e.g. diameter) $h$ and the time step size $\Delta t=t_{n}-t_{n-1}$. We apply regular subdivisions in time and space. Each edge of $T$ is divided into $m_{s}$ parts of equal length and the time interval is divided into $m_{t}$ parts (see Figure 3.1). We get $m_{t} \cdot m_{s}^{d}$ smaller prisms $\left\{\mathcal{Q}_{i}\right\}$ with spatial resolution $h / m_{s}$ and temporal resolution $\Delta t / m_{t}$.
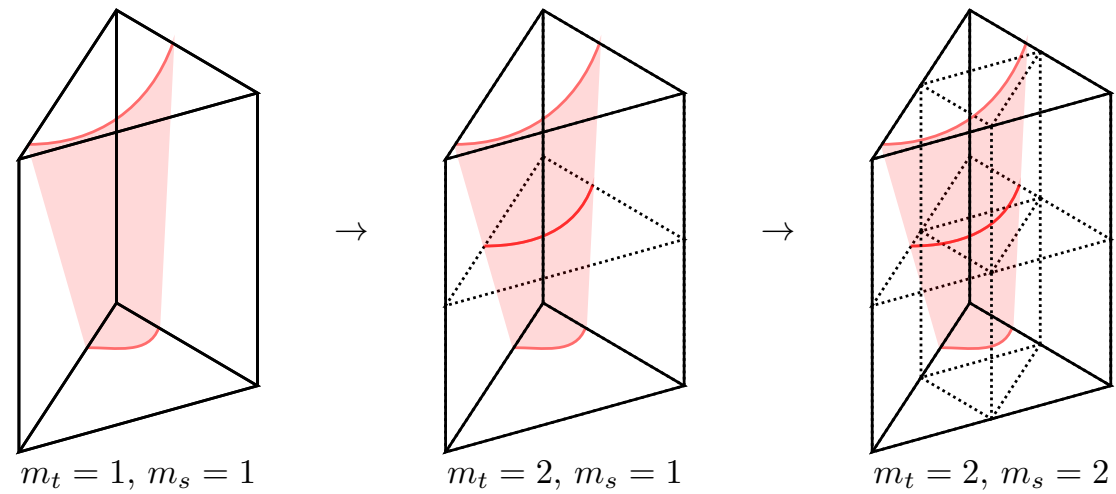

FIG. 3.1. An intersected prism in $d+1$ dimensions, with $d=2$. The original prism (left), the prisms after uniform subdivision in time (middle) and after subdivision in space and time (right).

Each (smaller) prism $\mathcal{Q}_{i}$ is subdivided into $d+1(d+1)$-simplices $\left\{\mathcal{P}_{j}\right\}$ (cf. section 5.2). On $\mathcal{P}_{j}$ the level-set function is interpolated as a linear function in space-time (by simply evaluating the vertex values only). As the level-set function is now represented as a linear function on each simplex, the according approximation of the zero-level of the level-set function is piecewise planar.

3.3. Finite elements. We briefly explain the construction of the basis functions for $W^{\Gamma_{*}}$. Let $\mathcal{J}^{W}=\left\{(i, k), i \in\left\{1, . ., N_{V}\right\}, k=1,2\right\}$ with $N_{V}$ the number of vertices of the spatial mesh denote the index set corresponding to the basis functions $q_{i, j}$ in $W$. Then the index set of basis functions in the space-time finite element space $W$ "close to the interface" is given by

$$
\mathcal{J}_{\Gamma_{*}}:=\left\{(i, k) \in \mathcal{J}^{W} \mid \operatorname{meas}_{d}\left(\Gamma_{*} \cap \operatorname{supp}\left(q_{i, k}\right)\right)>0\right\} .
$$


Let $H_{\Gamma_{*}}$ be the characteristic function corresponding to $Q_{2}$, i.e. $H_{\Gamma_{*}}(\mathbf{x}, t)=1$ if $(\mathbf{x}, t) \in Q_{2}$ and zero otherwise. For each space-time node index $(i, k) \in \mathcal{J}_{\Gamma_{*}}$ we have an additional basis function which is defined as follows:

$$
q_{i, k}^{\Gamma_{*}}(\mathbf{x}, t):=q_{i, k}(\mathbf{x}, t) \cdot\left(H_{\Gamma_{*}}(\mathbf{x}, t)-H_{\Gamma_{*}}\left(\mathbf{x}_{i}, t_{n-2+k}\right)\right), \quad(j, k) \in \mathcal{J}_{\Gamma_{*}}
$$

It is ensured that $q_{i, 1}^{\Gamma_{*}}\left(\mathbf{x}_{i}, t_{n-1}\right)=0, q_{i, 2}^{\Gamma_{*}}\left(\mathbf{x}_{i}, t_{n}\right)=0$ holds for all vertices $\mathbf{x}_{i}$. The characterization

$$
W^{\Gamma_{*}}=W \oplus \operatorname{span}\left\{q_{i, k}^{\Gamma_{*}} \mid(i, k) \in \mathcal{J}_{\Gamma_{*}}\right\}
$$

shows that the XFEM space $W^{\Gamma_{*}}$ is obtained by adding to the standard space $W$ new basis functions that are discontinuous across the space-time interface $\Gamma_{*}$.

Let the local index set of the local standard basis functions on one prism $\mathcal{Q}^{T}$ be $\mathcal{J}^{T}=\{(i, k), i=1, . ., 4, k=1,2\}$. The according shape functions are

$$
q_{i, k}(\mathbf{x}, t):=\phi_{i}(\mathbf{x}) \psi_{k}(t), \quad(i, k) \in \mathcal{J}^{T}
$$

with $\phi_{i}(\mathbf{x})=\lambda_{i}$ where $\lambda_{j}$ denotes the barycentric coordinate corresponding to vertex $j$ inside of tetrahedron $T$ and $\psi_{1}(t)=\frac{1}{\Delta t}\left(t^{n}-t\right), \psi_{2}(t)=\frac{1}{\Delta t}\left(t-t^{n-1}\right)$.

3.4. Numerical integration on non-intersected (space-time) volumes (case $(\mathrm{d}+1,0, \mathrm{n}))$. Whenever a (tetrahedral) element $T$ is not intersected by the interface $\Gamma(t)$ for the whole time interval $\left(t^{n-1}, t^{n}\right]$ the volume integrals of $a(\cdot, \cdot)$ and $f(\cdot)$ act on (the complete) prismatic element $\mathcal{Q}^{T}$. Consider for example the diffusion part on $\mathcal{Q}^{T}$. One corresponding matrix entry for $I=(i, k), J=(j, l) \in \mathcal{J}^{W}$ is $G_{I, J}^{T}:=g^{T}\left(q_{i, k}, q_{j, l}\right)$ with $g^{T}(u, v):=\int_{\mathcal{Q}^{T}} \alpha_{m} \beta_{m} \nabla u \nabla v d \mathbf{x} d t$ can be computed using iterated integrals:

$$
\begin{aligned}
g^{T}\left(q_{i, k}, q_{j, l}\right) & =\int_{t^{n-1}}^{t^{n}} \int_{T} \alpha_{m} \beta_{m}\left(\nabla \phi_{i}\right) \psi_{k}\left(\nabla \phi_{j}\right) \psi_{l} d \mathbf{x} d t \\
& =\int_{t^{n-1}}^{t^{n}} \psi_{k} \psi_{l} d t \int_{T} \alpha_{m} \beta_{m} \nabla \phi_{i} \nabla \phi_{j} d \mathbf{x}=\Delta t M_{k, l} \int_{T} \alpha_{m} \beta_{m} \nabla \phi_{i} \nabla \phi_{j} d \mathbf{x}
\end{aligned}
$$

where $\left\{M_{k, l}\right\}_{k, l=1,2}$ is the one-dimensional mass matrix

$$
M=\left(\begin{array}{cc}
1 / 3 & 1 / 6 \\
1 / 6 & 1 / 3
\end{array}\right)
$$

Thus, in this case the quadrature problem is reduced to a standard problem in $d$ dimensions. For the convection or r.h.s. term the velocity $\mathbf{w}$ and the source term $f$ are not necessarily separable (in terms of $t$ and $\mathbf{x}$ ). Nevertheless applying interpolation in time for $f$ and $\mathbf{w}$, e.g.

$$
f_{\Delta t}=f\left(t^{n-1}, x\right) \cdot \psi_{1}(t)+f\left(t^{n}, x\right) \cdot \psi_{2}(t)=\sum_{k=1}^{2} f\left(t^{k}, x\right) \cdot \psi_{k}(t)
$$

or tensor product quadrature rules, e.g.

$$
\begin{aligned}
h^{T}\left(q_{i, k}, q_{j, l}\right) & :=\int_{t^{n-1}}^{t^{n}} \int_{T} \beta_{m}\left(\nabla \phi_{i}\right) \psi_{k}\left(\mathbf{w} \cdot \nabla \phi_{j}\right) \psi_{l} d \mathbf{x} d t \\
& \approx \sum_{i=0}^{N_{i p}} \omega_{i} \psi_{k}\left(t_{i}\right) \psi_{l}\left(t_{i}\right) \int_{T} \beta_{m}\left(\nabla \phi_{i}\right)\left(\mathbf{w}\left(t_{i}\right) \cdot \nabla \phi_{j}\right) \psi_{l} d \mathbf{x} d t
\end{aligned}
$$


where $N_{i p}, t_{i}$ and $\omega_{i}$ are the information of the (1D) quadrature rule, the complexity of the quadrature problem is basically $d$-dimensional.

3.5. Numerical integration on intersected space-time volumes and the space-time interface $($ case $(d+1,0, c)$ and case $(d, 1, c))$. If $\mathcal{Q}^{T}$ is intersected some simplices $\mathcal{P}_{j}$ within the decomposition $\mathcal{Q}^{T}=\left\{\mathcal{P}_{j}\right\}$ (see section 3.2) are intersected by a planar approximation of the interface. Using the simplex and the (hyper-) plane one can find a decomposition of $\mathcal{P}_{j}$ into simplices $\left\{\mathcal{P}_{j}^{(k)}\right\}$ which are no longer intersected and form a decomposition of $\mathcal{P}_{j}, \mathcal{P}_{j}=\bigcup_{k} \mathcal{P}_{j}^{(k)}$. Furthermore the plane intersecting one simplex $\mathcal{P}_{j}$ can also be decomposed into uncut $d$-dimensional simplices. As this decomposition is neither obvious nor standard for the case $d=3$ a solution strategy is presented in detail in section 5. Thus one can achieve an explicit decomposition of $\mathcal{Q}_{m}^{T}$ into uncut $(d+1)$-dimensional simplices and of $\Gamma_{* h}$ into $d$-dimensional simplices. Once this decomposition is determined the integration can be applied simplex by simplex. Quadrature rules of high order for simplices can be found in standard references (see e.g. [24]) if the dimension of the simplex is $d+1 \leq 3$. For $d=3$, i.e. the simplex is four-dimensional (a pentatope) this is no longer standard. In the literature only a few integration rules can be found (see eg. [2] and [24]). In appendix A we quote lower order rules and strategies to generate higher order ones. Further in appendix B we comment on the computation of the weighting factor $\nu$.

3.6. Numerical integration on intersected space volumes. For the treatment of the cases $(d, 0, n)$ and $(d, 0, c)$ we refer to [9, Section 7.3].

4. Numerical examples. In this section we present results of numerical experiments. Different from the experiments in [20], where only spatially one-dimensional situations have been considered, we consider spatially three-dimensional cases to assess the convergence behavior of the method. In this setting we restrict ourselves to piecewise linear (in time and space) finite element approximations. In all examples we consider the $L^{2}(\Omega(T)$ )-error for different space and time resolutions. The time interval $[0, T]$ is divided into $n_{t}$ time slabs. The spatial domain is always a cube which is uniformly divided into $\left(n_{s}\right)^{d}$ smaller cubes which are then divided into tetrehedra. The error behaviour is investigated w.r.t. refinements, i.e. series of $n_{s}$ and $n_{t}$. For all computations we used a third order rule for the numerical integration on the (sub-)pentatopes (see also section A.2).

4.1. Moving plane, quasi-1D. This case is the three-dimensional counterpart to the one-dimensional test case in [20]. The domain is the cube $\Omega=[0,2]^{3}$. The "inner" phase is contained in the domain $\Omega_{1}(t)=\left\{\mathbf{x} \in \Omega:\left|x_{1}-q\left(x_{2}, x_{3}\right)-r(t)\right| \leq D / 2\right\}$, where $q:[0,2]^{2} \rightarrow[0,2]$ is the graph describing the shape of the domain $\Omega_{1}$ and $r:[0, T] \rightarrow \mathbb{R}$ the function describing the time-dependent shift of the interface in $x_{1}$-direction. $D=\frac{2}{3}$ is the width of the domain in $x_{1}$-direction. The complementary domain is $\Omega_{2}(t)=\Omega \backslash \Omega_{1}(t)$.

The velocity field is given as $\mathbf{w}=\left(\frac{\partial r}{\partial t}(t), 0,0\right)$. As boundary conditions we apply periodicity in all directions, $u\left(x_{i}=0\right)=u\left(x_{i}=2\right), i=1,2,3$. This renders the problem essentially one-dimensional if $q\left(x_{2}, x_{3}\right)=$ const.

We prescribe the r.h.s. source term $f$, such that the solution is given by

$$
u(\mathbf{x}, t)=\sin (k \pi t) \cdot U^{m}\left(x_{1}-q\left(x_{2}, x_{3}\right)-r(t)\right), \quad \mathbf{x} \in \Omega^{m}(t), \quad m=1,2
$$

with $U^{1}(y)=a y+b y^{3}$ and $U^{2}(y)=\sin (\pi y)$ where $a$ and $b$ are chosen such that the interface conditions hold. 

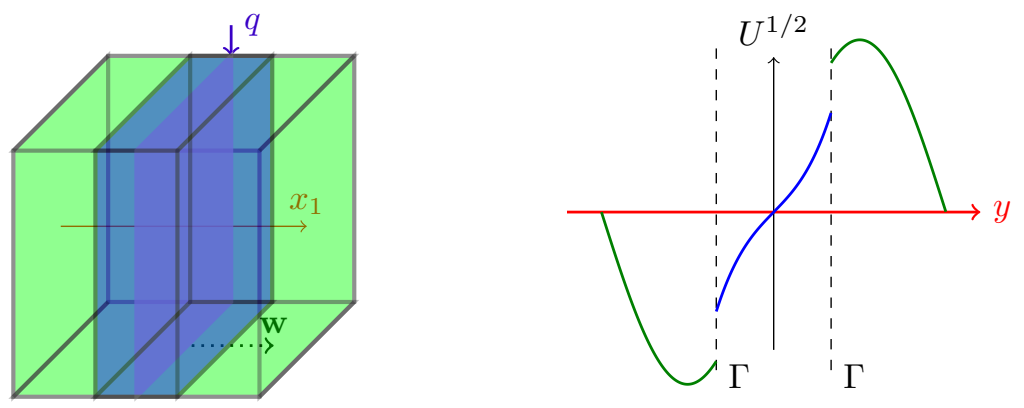

FIG. 4.1. Sketch of the geometrical setup of the numerical example in section 4.1 (left), $\Omega_{1}$ is blue and $\Omega_{2}$ is green. The time-independent part of the solution $U^{1 / 2}$ is also sketched (right).

The diffusivities are $\left(\alpha_{1}, \alpha_{2}\right)=(1,2)$ and the Henry weights $\left(\beta_{1}, \beta_{2}\right)=(1.5,1)$, resulting in $a \approx 1.02728$ and $b \approx 6.34294$. The problem is considered in the time interval $[0, T]$ with $T=1$.

4.1.1. Planar (in space and time) interface. We choose $q\left(x_{2}, x_{3}\right)=1$ and $r(t)=0.25 t$, hence the space-time interface is planar. Thus the proposed method for the approximation of the space-time interface is exact for every $m_{t}, m_{s} \geq 1$, where $m_{t}$ and $m_{s}$ are the number of subdivisions in time and each space direction, respectively, cf. section 3.2. We choose $m_{s}=m_{t}=1$.
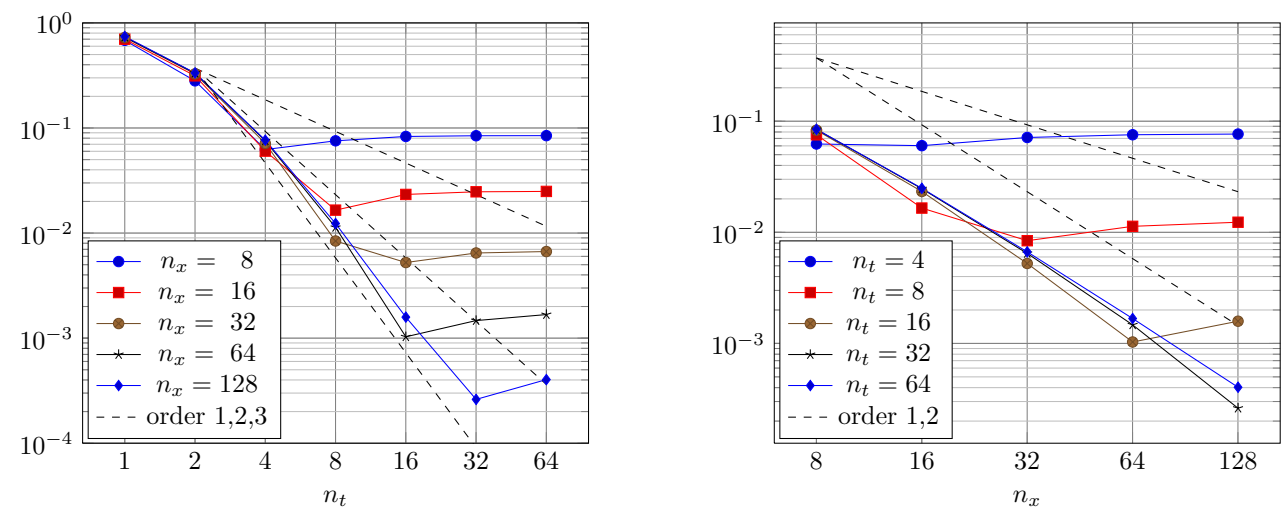

FIG. 4.2. Convergence in $L^{2}(\Omega(T))$-norm w.r.t. refinements in time (left) and space (right) for test case in section 4.1.1.

In Table 4.1 and Figure 4.2 we give the resulting error $\left\|u_{h}-u_{r e f}\right\|_{L^{2}(\Omega(T))}$ for different mesh and time step sizes. Similar to the results in [20] we observe a third order convergence w.r.t. time step size $\Delta t$ and a second order convergence w.r.t. the mesh size.

REMARK 4.1. In [25, Theorem 12.7] for the corresponding DG-FEM method applied to the standard heat equation an error bound with third order convergence w.r.t. $\Delta t$ has been derived. Note however that the analysis does not carry over for the case of the Nitsche-XFEM discretization.

4.1.2. Nonlinear moving interface. We consider $q\left(x_{2}, x_{3}\right)=\frac{7}{8}+\frac{1}{4} x_{2}^{2}\left(2-x_{2}\right)^{2}$ and $r(t)=\frac{1}{4 \pi} \sin (2 \pi t)$ which leads to a space-time interface which is no longer planar. The geometrical approximation of the space-time interface in this paper is piecewise planar, i.e. the maximum distance between $\Gamma_{*}$ and its approximation $\Gamma_{*, h}$ 


\begin{tabular}{|r|r|r|r|r|r|r|}
\hline$n_{t} \backslash n_{s}$ & 8 & 16 & 32 & 64 & 128 & eoc $_{t}$ \\
\hline 1 & 0.6826 & 0.7005 & 0.72760 & 0.73301 & 0.742317 & \\
2 & 0.2809 & 0.3138 & 0.32771 & 0.33260 & 0.334165 & 1.15 \\
4 & 0.0624 & 0.0603 & 0.07139 & 0.07557 & 0.076649 & 2.12 \\
8 & 0.0755 & 0.0165 & 0.00840 & 0.01132 & 0.012328 & 2.64 \\
16 & 0.0831 & 0.0233 & 0.00524 & 0.00103 & 0.001585 & 2.96 \\
32 & 0.0844 & 0.0247 & 0.00646 & 0.00147 & 0.000261 & 2.6 \\
\hline 64 & 0.0846 & 0.0249 & 0.00667 & 0.00168 & 0.000403 & -0.63 \\
\hline eoc $_{s}$ & \multicolumn{7}{|c|}{ TABLE 4.1 } & 1.76 & 1.90 & 1.99 & 2.06 & \\
\hline
\end{tabular}

$\left\|u_{h}-u_{r e f}\right\|_{L^{2}(\Omega(T))}$ for different refinements in time and space for test case in section 4.1.1. The last column shows the estimated order of convergence w.r.t. time (eoct) using the finest spatial resolution $\left(n_{s}=128\right)$, the last row shows the estimated order of convergence w.r.t. space (eoc $\left.)_{s}\right)$ using the finest temporal resolution $\left(n_{t}=64\right)$.

converges with second order w.r.t. increasing $n_{t} \cdot m_{t}, n_{s} \cdot m_{s}$. In Table 4.2 the error $\left\|u_{h}-u_{r e f}\right\|_{L^{2}(\Omega(T))}$ on a fixed (fine) spatial grid with resolution $64 \times 64 \times 64$ for different numbers of time steps is listed. In order to investigate the impact of the approximation of $\Gamma_{*}$ we performed the computation with different numbers of subdivisions $m_{s}, m_{t}$. The results, shown in Table 4.2, indicate an error bound behaviour of the form

\begin{tabular}{|r|r|r|r|r|r|r|r|}
\hline$n_{t}$ & 1 & 2 & 4 & 8 & 16 & 32 & 64 \\
\hline$m_{s}=1, m_{t}=1$ & 2.50 & 2.89 & 0.547 & 0.137 & 0.0342 & 0.00879 & 0.00317 \\
\hline eoc $_{t}$ & & -0.21 & 2.40 & 2.00 & 2.00 & 1.96 & 1.49 \\
\hline$m_{s}=1, m_{t}=2$ & 2.49 & 0.817 & 0.168 & 0.0374 & 0.00878 & 0.00301 & 0.00241 \\
\hline eoc $_{t}$ & & 1.61 & 2.28 & 2.17 & 2.09 & 1.54 & 0.32 \\
\hline$m_{s}=1, m_{t}=4$ & 0.520 & 0.481 & 0.0985 & 0.0167 & 0.00284 & 0.00219 & 0.00236 \\
\hline eoc $_{t}$ & & 0.11 & 2.29 & 2.56 & 2.56 & 0.37 & -0.11 \\
\hline$m_{s}=1, m_{t}=8$ & 0.491 & 0.412 & 0.0910 & 0.0143 & 0.00189 & 0.00212 & 0.00236 \\
\hline eoc $_{t}$ & & 0.25 & 2.18 & 2.67 & 2.92 & -0.17 & -0.15 \\
\hline$m_{s}=4, m_{t}=8$ & 0.491 & 0.412 & 0.0909 & 0.0142 & 0.00179 & 0.00207 & \\
\hline eoc $_{t}$ & & 0.25 & 2.18 & 2.68 & 2.99 & -0.20 & \\
\hline
\end{tabular}

Error $\left\|u_{h}-u_{r e f}\right\|_{L^{2}(\Omega(T))}$ for different temporal refinements and quadrature subdivisions on a regular $64 \times 64 \times 64$ tetrahedral mesh for the test case in section 4.1.2.

$$
\left\|u_{h}-u_{r e f}\right\|_{L^{2}(\Omega(T))} \leq C_{1} \Delta t^{3}+C_{2}\left(\Delta t / m_{t}\right)^{2}+C_{3}(h)
$$

where $C_{1}$ is independent of the approximation of $\Gamma_{*} . C_{2}$ is directly related to the interface approximation errors. If the interface approximation is exact (as in the last section) $C_{2}$ is zero. $C_{3}(h)$ describes the spatial error due to the method and the piecewise linear interface approximation for the numerical integration. In this examples $C_{3}(h) \approx 0.002$. Furthermore, we observe that in this example $C_{3}$ is essentially independent of $m_{s}$.

For $m_{t}$ sufficiently large, i.e. $m_{t}>\sqrt{C_{2} /\left(C_{1} \Delta t\right)}$ and $h$ sufficiently small, the first term dominates the error, such that one observes a third order in time convergence. This does not hold if $m_{t}$ is too small. Especially for $m_{s}=m_{t}=1$, the error is converging with (only) second order, due to a dominating interface approximation error.

REMARK 4.2. In order to investigate the additional effort within one time step due to additional XFEM unknowns, we consider the ratio between the maximum number of extended (XFEM) unknowns and standard (space-time) finite element unknowns within one time slab. In Figure 4.3 a sketch of the corresponding situation is shown. If 
the interface is well resolved, the number of unknowns close to the interface increases with $2^{d-1}$ for one uniform (spatial) refinement whereas the overall number of unknowns increases with $2^{d}$. Thus the ratio decreases linearly with the spatial resolution. In Table 4.3 the corresponding numbers for this test case are given which are in agreement with the expected behaviour.
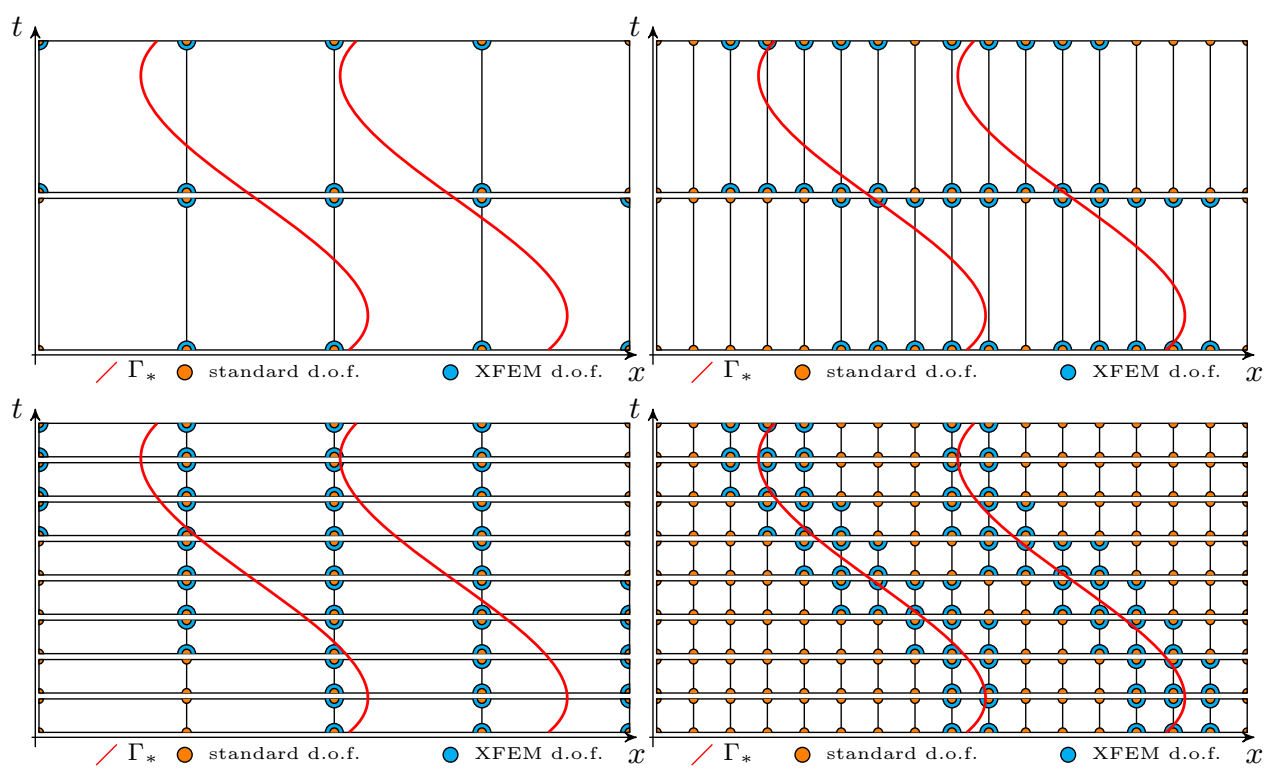

FIG. 4.3. Sketch of different spatial and temporal resolutions for a spatially one-dimensional problem. Standard and XFEM degrees of freedom are marked. For $n_{t}=2, n_{s}=4$ (upper left) the highest ratio between XFEM unknowns and standard unknowns within one time slab is 80\%, for $n_{t}=8, n_{s}=4$ (lower left) the ratio is also $80 \%$, for $n_{t}=2, n_{s}=16$ (upper right) the ratio is $59 \%$ and for $n_{t}=8, n_{s}=16$ (lower right) the ratio is $41 \%$.

\begin{tabular}{|r|r|r|r|r|r|}
\hline Std. unkn. & 1024 & 8192 & 65536 & 524288 & 4194304 \\
\hline$n_{t} \backslash n_{s}$ & 8 & 16 & 32 & 64 & 128 \\
\hline 1 & $736(72 \%)$ & $3648(45 \%)$ & $19328(30 \%)$ & $119808(23 \%)$ & $813056(19 \%)$ \\
2 & $656(64 \%)$ & $3008(37 \%)$ & $14336(22 \%)$ & $78848(15 \%)$ & $479232(11 \%)$ \\
4 & $656(64 \%)$ & $3008(37 \%)$ & $14336(22 \%)$ & $78848(15 \%)$ & $479232(11 \%)$ \\
8 & $656(64 \%)$ & $2880(35 \%)$ & $13056(20 \%)$ & $67072(13 \%)$ & $383488(9 \%)$ \\
16 & $640(63 \%)$ & $2624(32 \%)$ & $11136(17 \%)$ & $52992(10 \%)$ & $275456(7 \%)$ \\
32 & $640(63 \%)$ & $2496(30 \%)$ & $10368(16 \%)$ & $45824(9 \%)$ & $198656(5 \%)$ \\
64 & $640(60 \%)$ & $2496(30 \%)$ & $9856(15 \%)$ & $41472(8 \%)$ & $182784(4 \%)$ \\
\hline
\end{tabular}

Number of standard (space-time) unknowns (first row) and maximal number of additional XFEM unknowns for one time slab for different spatial and temporal resolutions for test case in section 4.1.2. In brackets the ratio between XFEM and standard unknowns is added.

REMARK 4.3. To decrease the (space-time) interface approximation error one can either choose smaller time steps or a larger refinement factor $m_{t}$ for the construction of $\Gamma_{*, h}$. The computation with a fixed $\Delta t=\Delta \tilde{t}$ and $m_{t}=\tilde{m}_{t}>1$ is cheaper than a computation with $\Delta t=\Delta \tilde{t} / \tilde{m}_{t}$ and $m_{t}=1$. In Figure 4.4 a sketch of both strategies is shown. For $m_{t}>1$ additional effort due to the decomposition strategy and quadrature within one time step is required. However if the interface is resolved, this is only 
required for a small number of elements (cf. Remark 4.2). The number of time steps and thereby the number of linear systems that have to be solved however is reduced by a factor of $\tilde{m}_{t}$. Note that the solution of linear systems is typically the most time consuming part.
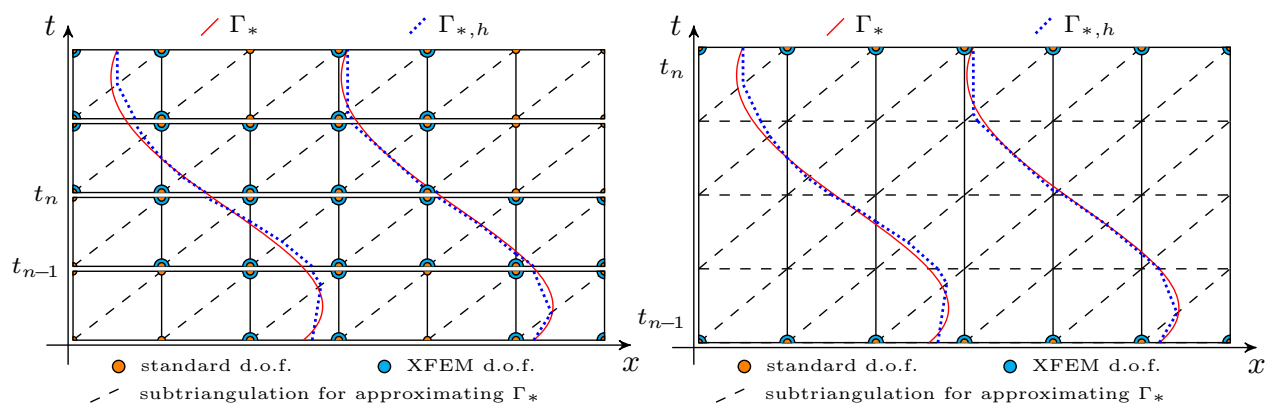

FIG. 4.4. Sketch of geometry approximation and degrees of freedom for the case $m_{t}=1$ and $\Delta t=\Delta \tilde{t} / 4$ (left) and for the case $m_{t}=4$ and $\Delta t=\Delta \tilde{t}$ (right). Note that the approximation quality of the piecewise planar interface is the same.

4.2. Rotational symmetric solution on a moving sphere. As a last example we consider a more realistic geometrical configuration. We consider a rotational symmetric solution for a stationary sphere and then translate it with a time-dependent velocity. The time interval is $[0, T]$ with $T=0.5$ and the domain is the cube $\Omega=[0,2]^{3}$. One phase is contained in the domain $\Omega_{1}(t)=\left\{\mathbf{x} \in \Omega:\left\|x-\left(\mathbf{p}_{0}+r(t) \cdot \mathbf{e}_{1}\right)\right\| \leq R\right\}$,
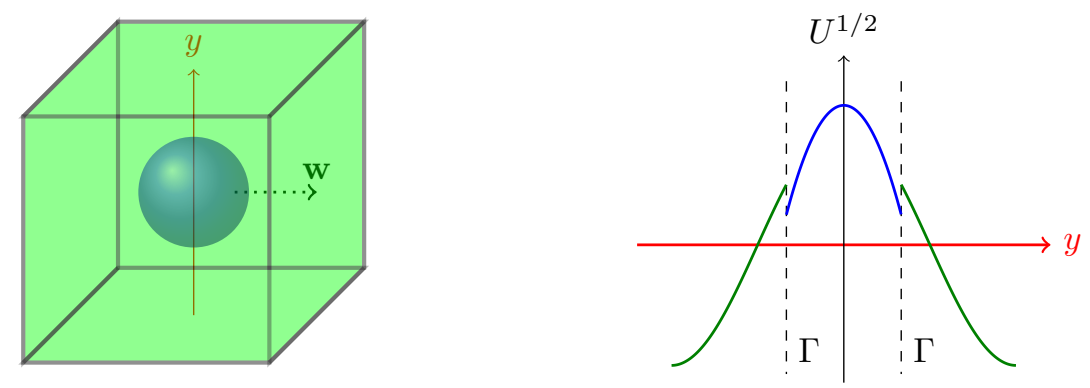

FIG. 4.5. Sketch of geometrical setup (left) for test case in section 4.2, $\Omega_{1}$ is blue and $\Omega_{2}$ is green. And sketch of the time-independent part of the solution $U^{1 / 2}$ (right).

where $\mathbf{p}_{0}$ is the center of the initial sphere and $r(t)$ the motion of the interface in $x_{1}$-direction, $\mathbf{e}_{1}$ is the corresponding unit vector. $R=\frac{1}{3}$ is the radius of the sphere. The complementary domain is $\Omega_{2}(t)=\Omega \backslash \Omega_{1}(t)$.

The velocity field $\mathbf{w}$ is given as $\mathbf{w}=\left(\frac{\partial r}{\partial t}(t), 0,0\right)$. As boundary conditions we apply suitable Dirichlet boundary conditions everywhere.

We prescribe these boundary conditions and the r.h.s. source term $f$, such that the solution is given by

$$
u(\mathbf{x}, t)=\sin (k \pi t) \cdot U^{m}\left(\left\|\mathbf{x}-\left(\mathbf{p}_{0}+r(t) \cdot \mathbf{e}_{1}\right)\right\|\right), \quad \mathbf{x} \in \Omega^{m}(t), \quad m=1,2
$$

with

$$
U^{1}(y)=a+b y^{2} \quad \text { and } \quad U^{2}(y)=\cos (\pi y)
$$


where $a$ and $b$ are chosen s.t. the interface conditions hold. The diffusivities are $\left(\alpha_{1}, \alpha_{2}\right)=(10,20)$ and the Henry weights $\left(\beta_{1}, \beta_{2}\right)=(2,1)$ resulting in $a \approx 1.1569$ and $b \approx-8.1621$. The problem is considered in the time interval $[0, T]$ with $T=0.5$. We choose $\mathbf{p}_{0}=(0.5,1,1)^{T}$ and $r(t)=\frac{1}{4 \pi} \sin (2 \pi t)$. For the approximation of the space-time interface we consider $m_{s}=m_{t}=1$. We observe an error behaviour which is of (at least) second order in time and space $\left(\mathcal{O}\left(h^{2}+\Delta t^{2}\right)\right)$ (see Figure 4.6). For the finest spatial resolution $\left(n_{s}=64\right)$ we observe an order around 2.5 for the convergence in time. In contrast to the previous test cases the spatial error dominates the overall error already for coarse temporal resolutions. We expect that for finer spatial resolutions and better geometry approximations $\left(m_{t}>1, m_{s} \geq 1\right)$ one could retain the third order convergence in time.

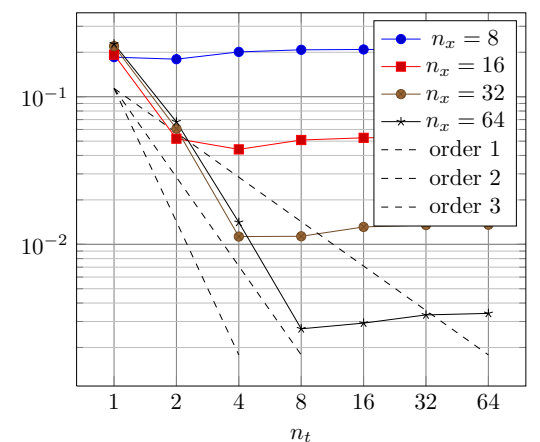

\begin{tabular}{|c|r|r|r|r|}
\hline$n_{t} \backslash n_{s}$ & 8 & 16 & 32 & 64 \\
\hline 1 & 0.185 & 0.1929 & 0.2202 & 0.22794 \\
2 & 0.180 & 0.0520 & 0.0607 & 0.06760 \\
4 & 0.201 & 0.0440 & 0.0113 & 0.01409 \\
8 & 0.208 & 0.0509 & 0.0113 & 0.00268 \\
16 & 0.209 & 0.0527 & 0.0131 & 0.00293 \\
32 & 0.209 & 0.0530 & 0.0135 & 0.00332 \\
64 & 0.209 & 0.0530 & 0.0136 & 0.00340 \\
\hline
\end{tabular}

Fig. 4.6. Convergence in $L^{2}(\Omega(T))$-norm w.r.t. refinements in time and space as a plot and as tabulated values for test case in section 4.2.

It is also relevant to study the accuracy of the method w.r.t. the interface condition. Therefore, in Table 4.4 we consider the error

$$
\left\|\nu^{\frac{1}{2}}\left[\beta u_{h}\right]\right\|_{L^{2}\left(\Gamma_{*, h}\right)}=\left(\int_{0}^{T} \int_{\Gamma_{h}(t)}\left[\beta u_{h}\right]^{2} d s d t\right)^{\frac{1}{2}}
$$

under space and time refinement and also observe an $\mathcal{O}\left(h^{2}+\Delta t^{2}\right)$ behaviour.

\begin{tabular}{|c|c|c|c|c|c|}
\hline$n_{t} \backslash n_{s}$ & 8 & 16 & 32 & 64 & $\mathrm{eoc}_{t}$ \\
\hline 2 & 0.0495 & 0.00700 & 0.0198 & 0.0587 & \\
\hline 4 & 0.0430 & 0.00384 & 0.00567 & 0.0227 & 1.37 \\
\hline 8 & 0.0417 & 0.00253 & 0.00164 & 0.00517 & 2.13 \\
\hline 16 & 0.0414 & 0.00205 & 0.000716 & 0.00117 & 2.14 \\
\hline 32 & 0.0413 & 0.00190 & 0.000523 & 0.000275 & 2.10 \\
\hline 64 & 0.0413 & 0.00186 & 0.000477 & 0.000131 & 1.07 \\
\hline $\mathrm{eoc}_{s}$ & & 4.48 & 1.96 & 1.86 & \\
\hline
\end{tabular}

Interface error $\left\|\nu^{\frac{1}{2}}\left[\beta u_{h}\right]\right\|_{L^{2}\left(\Gamma_{*, h}\right)}$ for different refinements in time and space for the test case in section 4.2. The last column shows the estimated order of convergence w.r.t. time (eoct) using the finest spatial resolution $\left(n_{s}=64\right)$, the last row shows the estimated order of convergence w.r.t. space (eocs) using the finest temporal resolution $\left(n_{t}=64\right)$.

5. A strategy to decompose intersected 4-prisms into pentatopes. In this section we introduce a decomposition strategy that allows for a decomposition of four dimensional prisms into pentatopes as needed in section 3.5. This approach is new. 
Firstly, we introduce the definitions of relevant four dimensional geometries in section 5.1. The decomposition of a 4-prism into four pentatopes is presented in section 5.2. This is already needed to construct (via interpolation of the level-set function) the piecewise planar space-time interface in section 3.2.

In section 5.4 a strategy is presented that allows us to decompose a pentatope which is intersected by a hyperplane (representing an approximation of the space-time interface) into pentatopes which are not intersected. Figure 5.1 sketches the algorithmic structure of the decomposition strategy. In this algorithm we need a particular geometrical object, that we call hypertriangle, which can be decomposed into six pentatopes following the decomposition rule in section 5.3.

REMARK 5.1. The resulting pentatopes/tetrahedra in this decomposition can have arbitrary small angles. Note that this does not lead to stability problems as we are using the decomposition only for numerical integration.

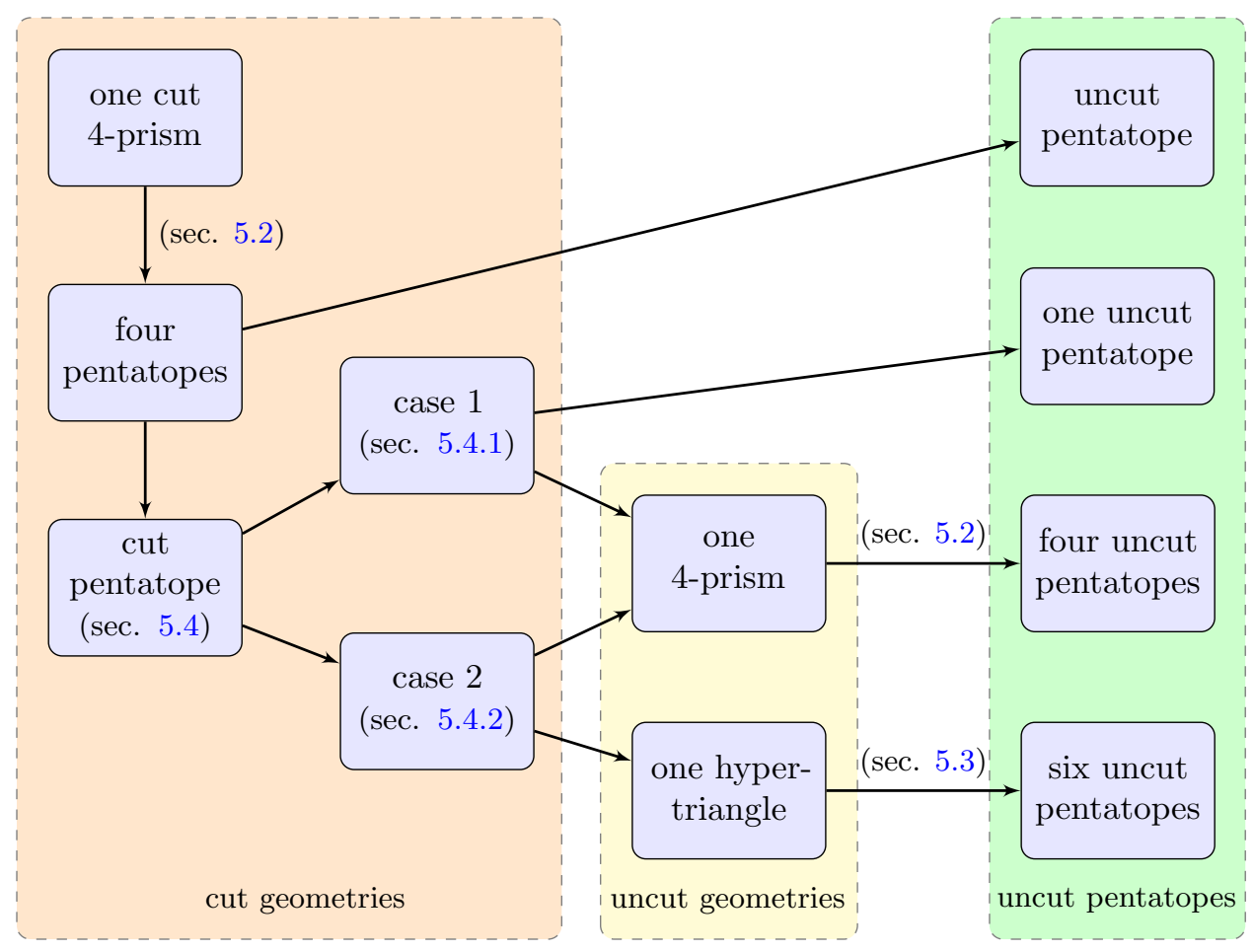

FIG. 5.1. Algorithmic structure of the decomposition strategy proposed in section 5.

5.1. Definition of simple geometries in four dimensions. By $\mathbf{e}^{i} \in \mathbb{R}^{n}$ we denote the $i$-th unit vector with $\left(\mathbf{e}^{i}\right)_{j}=\delta_{i, j}$ for $i=1, . ., n$ and $\mathbf{e}^{0}:=0$.

Definition 5.1 (4-simplex / pentatope). Let $\mathbf{x}^{i} \in \mathbb{R}^{4}$ for $i=1, . ., 5$ and $\mathbf{d}^{i, j}:=\mathbf{x}^{i}-\mathbf{x}^{j}$. Iff the vectors $\mathbf{d}^{i, 1}$ for $i=2, . ., 5$ are linearly independent, we call the convex hull $\mathcal{P}=\operatorname{conv}\left(\left\{\mathrm{x}^{i}\right\}_{i=1, . .5}\right)$ the 4 -simplex or pentatope.

REMARK 5.2 (reference pentatope). Every pentatope $\mathcal{P}$ can be represented as an affine transformation applied to the reference pentatope $\hat{\mathcal{P}}=\operatorname{conv}\left(\left\{\mathbf{e}^{i}\right\}_{i=0, . .4}\right)$. The transformation has the form

$$
\Phi: \hat{\mathcal{P}} \rightarrow \mathcal{P},\left(\hat{x}_{1}, \hat{x}_{2}, \hat{x}_{3}, \hat{x}_{4}\right) \rightarrow \sum_{i=1}^{5} \hat{\lambda}_{i} \mathbf{x}^{i},
$$


where $\hat{\lambda}_{i}\left(\hat{x}_{1}, \hat{x}_{2}, \hat{x}_{3}, \hat{x}_{4}\right)$ are the barycentric coordinates of $\hat{\mathcal{P}}$ w.r.t. vertex $\mathbf{e}^{i-1}$. Definition 5.2 (4-prism). Let $\mathbf{x}^{i} \in \mathbb{R}^{4}$ for $i=1, . ., 4$ and $\mathbf{y} \in \mathbb{R}^{4}$. Iff $\left\{\mathbf{x}^{i}\right\}_{i=1, \ldots, 4}$ defines a 3-simplex (tetrahedron) $T=\operatorname{conv}\left(\left\{\mathbf{x}^{i}\right\}_{i=1, . .4}\right)$ and $\mathbf{y}$ is linearly independent of $\left\{\mathbf{d}^{i, 1}\right\}_{i=2, . .4}$, with $\mathbf{d}^{i, j}:=\mathbf{x}^{i}-\mathbf{x}^{j}$, the set

$$
\mathcal{Q}=\operatorname{conv}\left(\left\{\mathbf{x}^{i}\right\}_{i=1, . ., 4},\left\{\mathbf{x}^{i}+\mathbf{y}\right\}_{i=1, . ., 4}\right)=\left\{\mathbf{x}+\alpha \mathbf{y}, \mathbf{x} \in \operatorname{conv}\left(\left\{\mathbf{x}^{i}\right\}_{i=1, . .4}\right), \alpha \in[0,1]\right\}
$$

is called 4-prism.

REMARK 5.3 (reference 4-prism). Every 4-prism can be represented as an affine linear transformation applied to the reference 4-prism $\hat{\mathcal{Q}}=\operatorname{conv}\left(\left\{\mathbf{e}^{i}\right\}_{i=0, . ., 3}\right\},\left\{\mathbf{e}^{i}+\right.$ $\left.\left.\left.\mathbf{e}^{4}\right\}_{i=0, . ., 3}\right\}\right)$. The transformation has the form

$$
\Phi: \hat{\mathcal{Q}} \rightarrow \mathcal{Q},\left(\hat{x}_{1}, \hat{x}_{2}, \hat{x}_{3}, \hat{x}_{4}\right) \rightarrow \sum_{i=1}^{4} \hat{\mu}_{i} \mathbf{x}^{i}+\hat{x}_{4} \mathbf{y}
$$

where $\hat{\mu}_{i}\left(\hat{x}_{1}, \hat{x}_{2}, \hat{x}_{3}\right)$ are the barycentric coordinates of the reference tetrahedron $\hat{T}=$ $\operatorname{conv}\left(\left\{\mathbf{e}^{i}\right\}_{i=0, . .3}\right)$. The next geometry is a little bit more complex. It later occurs as
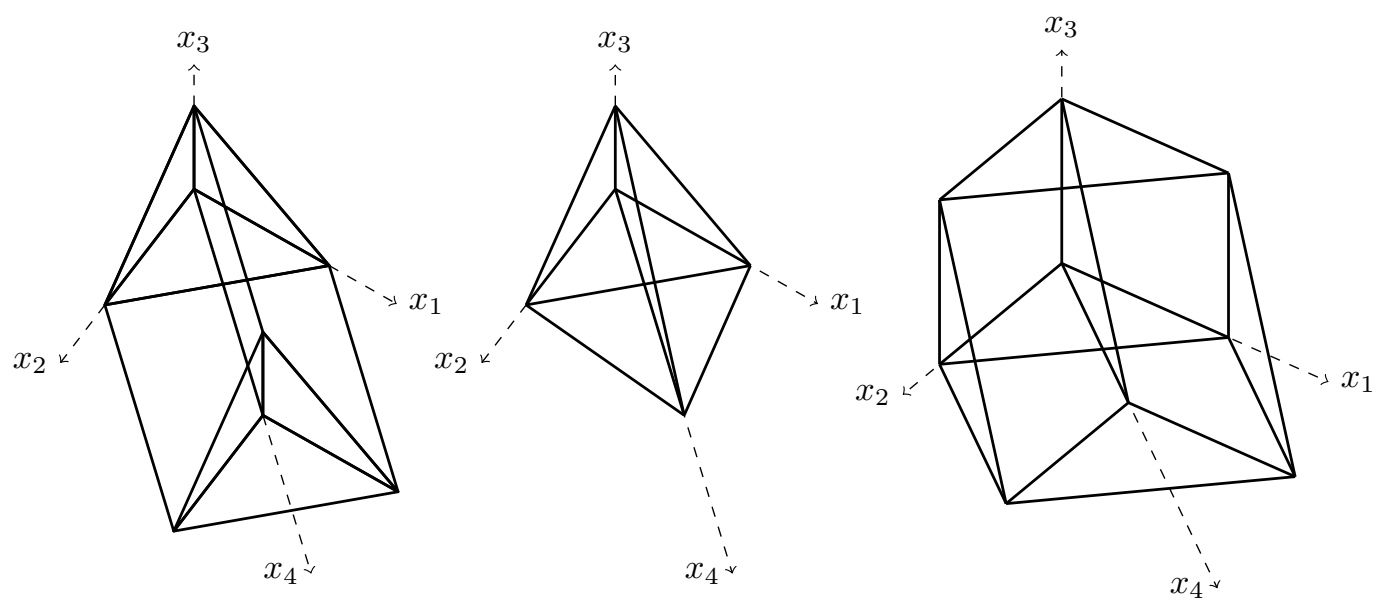

FIG. 5.2. Sketch of reference geometries. Reference 4-prism $\hat{\mathcal{Q}}$ (left), reference pentatope $\hat{\mathcal{P}}$ (center) and reference hypertriangle $\hat{\mathcal{H}}$ (right).

one part of a pentatope cut by a hyperplane.

DeFinition 5.3 (hypertriangle). We define the reference hypertriangle as

$$
\begin{aligned}
\hat{\mathcal{H}} & :=\left\{\left(x_{1}, x_{2}, x_{3}, x_{4}\right) \in \mathbb{R}_{+}^{4}, x_{1}+x_{2} \leq 1, x_{3}+x_{4} \leq 1\right\} \\
& =\operatorname{conv}\left(\left\{\hat{\mathbf{x}}^{i, j}\right\}_{i=1, . ., 3, j=1, . ., 3}\right)=\hat{\mathcal{K}} \times \hat{\mathcal{K}}
\end{aligned}
$$

where $\hat{\mathcal{K}} \subset \mathbb{R}^{2}$ denotes the reference triangle $\hat{\mathcal{K}}=\operatorname{conv}\left(\left\{\chi^{1}, \chi^{2}, \chi^{3}\right\}\right) \subset \mathbb{R}^{2}$ with $\chi^{i}=\mathbf{e}^{i-1} \in \mathbb{R}^{2}, i=1,2,3$ and $\hat{\mathbf{x}}^{i, j}=\left(\chi^{i}, \chi^{j}\right) \in \mathbb{R}^{4}, i, j=1,2,3$. Now, let $\mathbf{x}^{i, j} \in$ $\mathbb{R}^{4}, i, j=1,2,3$. The convex hull $\mathcal{H}=\operatorname{conv}\left(\left\{\mathbf{x}^{i, j}\right\}_{i, j=1,2,3}\right)$ is called a hypertriangle iff there exists a transformation

$$
\Phi: \hat{\mathcal{H}} \rightarrow \mathbb{R}^{4},\left(\hat{x}_{1}, \hat{x}_{2}, \hat{x}_{3}, \hat{x}_{4}\right) \rightarrow \sum_{i=1}^{3} \sum_{j=1}^{3} \hat{\rho}_{i}\left(\hat{x}_{1}, \hat{x}_{2}\right) \hat{\rho}_{j}\left(\hat{x}_{3}, \hat{x}_{4}\right) \mathbf{x}^{i, j}
$$

where $\hat{\rho}_{i}\left(\hat{x}_{1}, \hat{x}_{2}\right)$ is the barycentric coordinate of the reference triangle $\hat{\mathcal{K}}$ corresponding to the vertex $\chi^{i}$. There holds $\Phi(\hat{\mathcal{H}})=\mathcal{H}$. 
5.2. Decomposition of a 4-prism into four pentatopes. We consider an arbitrary prism element $\mathcal{Q}^{T}=T \times I_{n}$ with a tetrahedral element $T$ and a time interval $I_{n}$. For each $\mathcal{Q}^{T}$ there exists a linear transformation $\Phi$ mapping from the reference 4-prism $\hat{\mathcal{Q}}$ to $\mathcal{Q}^{T}$ which is of the form $\Phi(\hat{\mathbf{x}}, \hat{t})=\left(\Phi_{x}(\hat{\mathbf{x}}), \Phi_{t}(\hat{t})\right)^{T}$ with the time transformation $\Phi_{t}(\hat{t})=\hat{t} \cdot t_{n}+(1-\hat{t}) \cdot t_{n-1}$ and the space transformation $\Phi_{x}(\hat{\mathbf{x}})$ mapping from the reference tetrahedron $T$ to $T$.

It is sufficient to consider the decomposition of the reference 4-prism $\hat{\mathcal{Q}}$ into four pentatopes as applying $\Phi$ to each pentatope of this decomposition results in a valid decomposition of $\mathcal{Q}$ into four pentatopes. With $\mathrm{x}^{i}:=\mathbf{e}^{i-1}$ and $\mathbf{y}^{i}:=\mathbf{e}^{i-1}+\mathbf{e}^{4}$ for $i=1, . ., 4$ for the reference 4 -prism there holds $\hat{\mathcal{Q}}=\operatorname{conv}\left(\left\{\mathbf{x}^{i}\right\}_{i=1, . .4},\left\{\mathbf{y}^{i}\right\}_{i=1, . .4}\right)$. We decompose $\hat{\mathcal{Q}}$ into four pentatopes $\hat{\mathcal{P}}_{1}, \hat{\mathcal{P}}_{2}, \hat{\mathcal{P}}_{3}, \hat{\mathcal{P}}_{4}$, which are defined as follows:

$$
\begin{aligned}
& \hat{\mathcal{P}}_{1}:=\operatorname{conv}\left(\left\{\mathrm{x}^{1}, \mathrm{x}^{2}, \mathrm{x}^{3}, \mathrm{x}^{4}, \mathrm{y}^{4}\right\}\right), \hat{\mathcal{P}}_{2}:=\operatorname{conv}\left(\left\{\mathrm{x}^{1}, \mathrm{x}^{2}, \mathrm{x}^{3}, \mathrm{y}^{3}, \mathrm{y}^{4}\right\}\right) \\
& \hat{\mathcal{P}}_{3}:=\operatorname{conv}\left(\left\{\mathrm{x}^{1}, \mathrm{x}^{2}, \mathrm{y}^{2}, \mathrm{y}^{3}, \mathrm{y}^{4}\right\}\right), \hat{\mathcal{P}}_{4}:=\operatorname{conv}\left(\left\{\mathrm{x}^{1}, \mathrm{y}^{1}, \mathrm{y}^{2}, \mathrm{y}^{3}, \mathrm{y}^{4}\right\}\right)
\end{aligned}
$$

A sketch of those can be found in Figure 5.3. One can easily show that the pentatopes

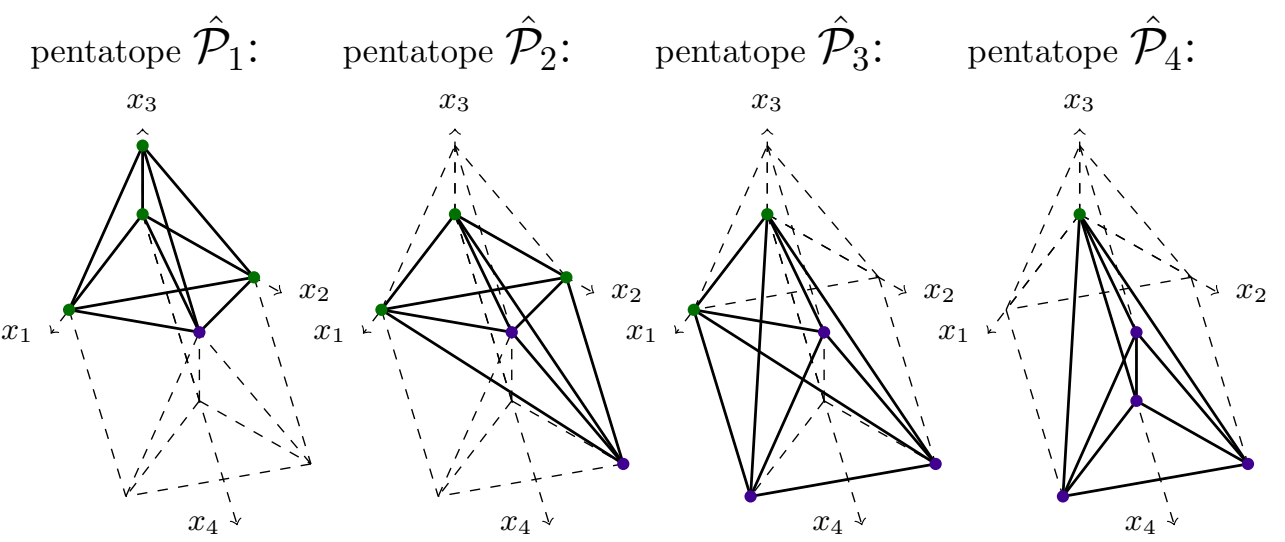

FIG. 5.3. Sketch of pentatopes $\hat{\mathcal{P}}_{1}, \hat{\mathcal{P}}_{2}, \hat{\mathcal{P}}_{3}, \hat{\mathcal{P}}_{4}$ which form a valid decomposition of the reference 4-prism $\hat{\mathcal{Q}}$.

are disjoint (except for a part with measure zero) and sum up to the reference prism: $\bigcup \hat{\mathcal{P}}_{i}=\hat{\mathcal{Q}}$. Note further that the measure of all pentatopes are the same, i.e. $\operatorname{meas}_{4}\left(\mathcal{P}_{i}\right)=1 / 24$.

5.3. Decomposing the reference hypertriangle. Let $\mathbf{u}^{i}=\hat{\mathbf{x}}^{1, i}, \mathbf{v}^{i}=\hat{\mathbf{x}}^{2, i}$, $\mathbf{w}^{i}=\hat{\mathbf{x}}^{3, i}, i=1, . ., 3$ with $\hat{\mathbf{x}}^{i, j}$ as in definition 5.3. We decompose $\hat{\mathcal{H}}$ into six pentatopes which are defined as follows:

$$
\begin{array}{ll}
\hat{\mathcal{D}}_{u}=\operatorname{conv}\left(\left\{\underline{\mathbf{u}}^{1}, \underline{\mathbf{u}}^{2}, \underline{\mathbf{u}}^{3}, \mathbf{v}^{2}, \mathbf{w}^{3}\right\}\right), & \hat{\mathcal{D}}_{v}=\operatorname{conv}\left(\left\{\mathbf{u}^{1}, \underline{\mathbf{v}}^{1}, \mathbf{v}^{2}, \mathbf{v}^{3}, \mathbf{w}^{3}\right\}\right) \\
\hat{\mathcal{D}}_{w}=\operatorname{conv}\left(\left\{\mathbf{u}^{1}, \mathbf{v}^{2}, \underline{\mathbf{w}}^{1}, \mathbf{w}^{2}, \underline{\mathbf{w}}^{3}\right\}\right), & \hat{\mathcal{D}}_{1}=\operatorname{conv}\left(\left\{\underline{\mathbf{u}}^{1}, \mathbf{v}^{1}, \mathbf{v}^{2}, \mathbf{w}^{1}, \mathbf{w}^{3}\right\}\right) \\
\hat{\mathcal{D}}_{2}=\operatorname{conv}\left(\left\{\mathbf{u}^{1}, \underline{\mathbf{u}}^{2}, \underline{\mathbf{v}}^{2}, \underline{\mathbf{w}}^{2}, \mathbf{w}^{3}\right\}\right), & \hat{\mathcal{D}}_{3}=\operatorname{conv}\left(\left\{\underline{\mathbf{u}}^{1}, \underline{\mathbf{u}}^{3}, \mathbf{v}^{2}, \underline{\mathbf{v}}^{3}, \underline{\mathbf{w}}^{3}\right\}\right)
\end{array}
$$

Note that there is a simple structure behind this decomposition. We define the "diagonal triangle" as $\hat{K}_{\text {diag }}=\operatorname{conv}\left(\mathbf{u}^{1}, \mathbf{v}^{2}, \mathbf{w}^{3}\right)$. To the three vertices of $\hat{K}_{\text {diag }}$ we add the missing vertices (underlined) of one of the following six triangles

$$
\begin{array}{lll}
\hat{K}_{u}=\operatorname{conv}\left(\left\{\mathbf{u}^{1}, \mathbf{u}^{2}, \mathbf{u}^{3}\right\}\right), & \hat{K}_{v}=\operatorname{conv}\left(\left\{\mathbf{v}^{1}, \mathbf{v}^{2}, \mathbf{v}^{3}\right\}\right), & \hat{K}_{w}=\operatorname{conv}\left(\left\{\mathbf{w}^{1}, \mathbf{w}^{2}, \mathbf{w}^{3}\right\}\right), \\
\hat{K}_{1}=\operatorname{conv}\left(\left\{\mathbf{u}^{1}, \mathbf{v}^{1}, \mathbf{w}^{1}\right\}\right), & \hat{K}_{2}=\operatorname{conv}\left(\left\{\mathbf{u}^{2}, \mathbf{v}^{2}, \mathbf{w}^{2}\right\}\right), & \hat{K}_{3}=\operatorname{conv}\left(\left\{\mathbf{u}^{3}, \mathbf{v}^{3}, \mathbf{w}^{3}\right\}\right) .
\end{array}
$$



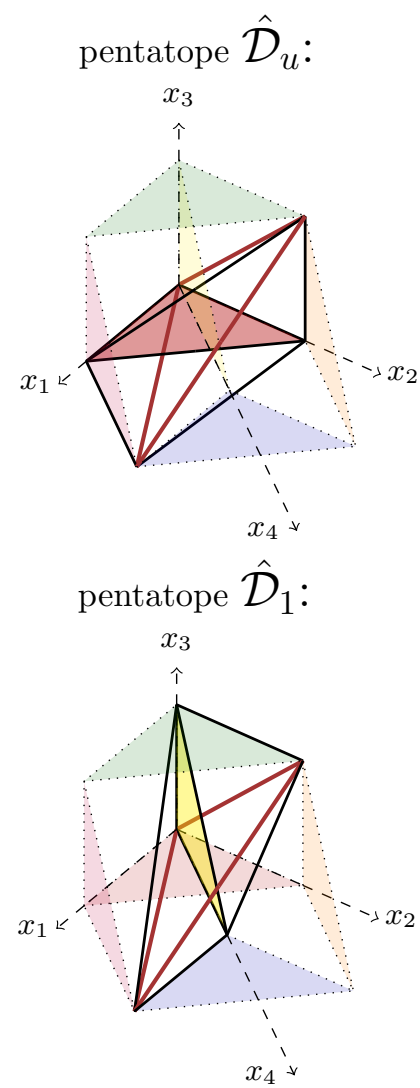

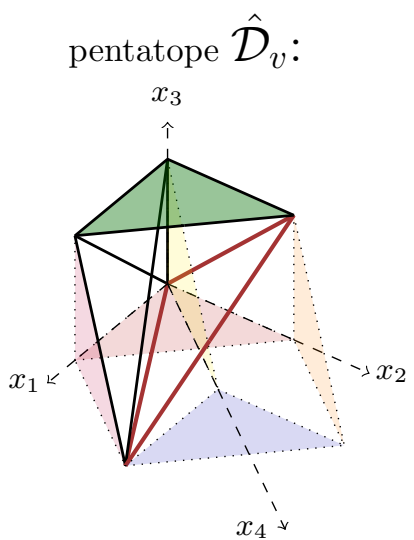

pentatope $\hat{\mathcal{D}}_{2}$ :

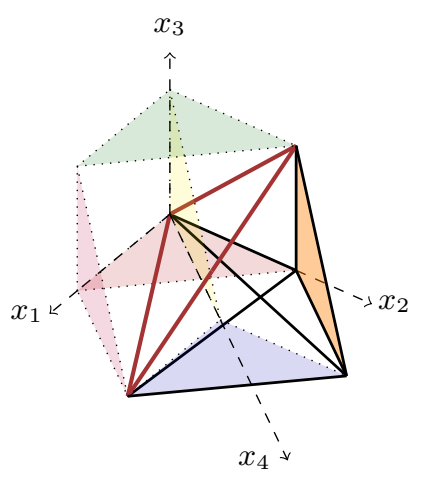

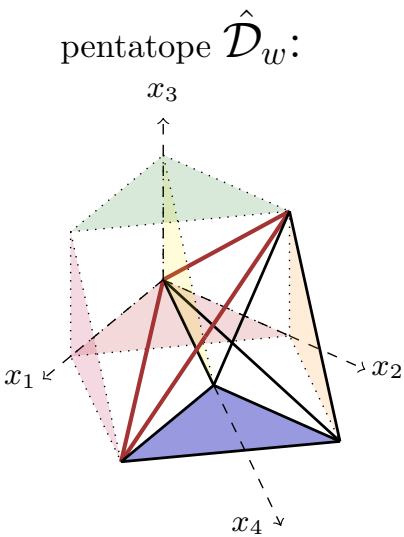

pentatope $\hat{\mathcal{D}}_{3}$ :

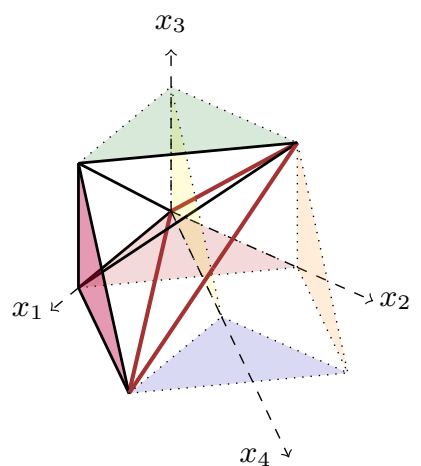

FIG. 5.4. Sketch of pentatopes $\hat{\mathcal{D}}_{u}, \hat{\mathcal{D}}_{v}, \hat{\mathcal{D}}_{w}, \hat{\mathcal{D}}_{1}, \hat{\mathcal{D}}_{2}, \hat{\mathcal{D}}_{3}$ which form a valid decomposition of the reference hypertriangle $\hat{\mathcal{H}}$. The edges of $\hat{K}_{\text {diag }}$ are highlighted in red, whereas the triangles $\hat{K}_{u}, \hat{K}_{v}, \hat{K}_{w}, \hat{K}_{1}, \hat{K}_{2}, \hat{K}_{3}$ are filled with the corresponding color. The triangle corresponding to each pentatope is highlighted especially.

A sketch of those pentatopes is given in Figure 5.4. Also here, one can easily show that the pentatopes are disjoint (except for a part with measure zero), and sum up to $\hat{\mathcal{H}}$.

5.4. Decomposition of a pentatope intersected by the space-time interface. We assume that the space-time interface is approximated in a piecewise planar fashion, s.t. within each pentatope the space-time interface is a (hyper-)plane. This plane divides a pentatope into two parts. Note that due to the pentatope being a convex set each of the two parts will still be convex. We now consider a pentatope $\mathcal{P}$ which is cut by the plane $\mathcal{G}=\left\{\mathbf{x} \in \mathbb{R}^{4}: \mathbf{x} \cdot \mathbf{n}_{\mathcal{G}}=c\right\}$ which represents the local approximation of the space-time interface. Each vertex $\mathbf{v}$ is marked corresponding to one of the two halfspaces. Vertices with $\mathbf{v} \cdot \mathbf{n}_{\mathcal{G}}<c$ are marked with a plus $(+)$, all others with a minus (-). Note that this classification includes the cases where the space-time interface hits vertices $\left(\mathbf{v} \cdot \mathbf{n}_{\mathcal{G}}=c\right)$. We thus can only have two non-trivial situations:

Case 1: One vertex has a sign that is different from all the others or

Case 2: Two vertices have a sign that is different from the other three vertices.

In the following we will consider these cases separately and construct a decomposition of the parts into pentatopes. Without loss of generality we assume that the vertices in 
the smaller group of vertices are those marked with a plus $(+)$.

5.4.1. Case 1: Decomposition into one pentatope and one 4-prism. We consider the case where one vertex of a pentatope, say $\mathbf{x}^{5}$, is marked with a plus $(+)$. All other vertices $\left(\mathbf{x}^{1}, \mathbf{x}^{2}, \mathbf{x}^{3}, \mathbf{x}^{4}\right)$ are marked with a minus (-). The cutting points of the hyperplane $\mathcal{G}$ with the edges are $\mathbf{b}^{1}:=\overline{\mathbf{x}^{1} \mathbf{x}^{5}} \cap \mathcal{G}, \mathbf{b}^{2}:=$ $\overline{\mathbf{x}^{2} \mathbf{x}^{5}} \cap \mathcal{G}, \mathbf{b}^{3}:=\overline{\mathbf{x}^{3} \mathbf{x}^{5}} \cap \mathcal{G}, \mathbf{b}^{4}:=\overline{\mathbf{x}^{4} \mathbf{x}^{5}} \cap \mathcal{G}$. The geometry containing the separated vertex is the pentatope $\mathcal{P}^{+}:=\operatorname{conv}\left(\left\{\mathbf{b}^{1}, \mathbf{b}^{2}, \mathbf{b}^{3}, \mathbf{b}^{4}, \mathbf{x}^{5}\right\}\right)$ while the remainder is $\mathcal{Q}^{-}:=\operatorname{conv}\left(\left\{\mathbf{x}^{1}, \mathbf{x}^{2}, \mathbf{x}^{3}, \mathbf{x}^{4}, \mathbf{b}^{1}, \mathbf{b}^{2}, \mathbf{b}^{3}, \mathbf{b}^{4}\right\}\right)$. Consider the mapping

$$
\Phi: \hat{\mathcal{Q}} \rightarrow \mathcal{Q}^{-},\left(\hat{x}_{1}, \hat{x}_{2}, \hat{x}_{3}, \hat{x}_{4}\right) \rightarrow \sum_{i=1}^{4} \mu_{i}\left(\hat{x}_{1}, \hat{x}_{2}, \hat{x}_{3}\right)\left(\hat{x}_{4} \mathbf{b}^{i}+\left(1-\hat{x}_{4}\right) \mathbf{x}^{i}\right)
$$

with $\hat{\mu}\left(\hat{x}_{1}, \hat{x}_{2}, \hat{x}_{3}\right)$ the barycentric coordinates of the reference tetrahedron $\hat{T}$. The decomposition of the reference 4 -prism $\hat{\mathcal{Q}}$ into the four pentatopes $\hat{\mathcal{P}}_{i}, i=1, . ., 4$ as described in section 5.2 can be used as a triangulation of $\hat{\mathcal{Q}}$. Let $\Phi_{h}$ be the (pentatope-) piecewise linear interpolation of $\Phi$ at the vertices of this triangulation. One can show that $\Phi_{h}$ is an isomorphism between $\hat{\mathcal{Q}}$ and $\mathcal{Q}^{-}$and furthermore for each pentatope $\hat{\mathcal{P}}_{i}$ the image $\Phi_{h}\left(\hat{\mathcal{P}}_{i}\right)$ is again a pentatope. Thus the decomposition rule for the reference 4 -prism can also be applied here and we get a valid decomposition by taking the four pentatopes

$$
\begin{gathered}
\mathcal{P}_{1}=\Phi_{h}\left(\hat{\mathcal{P}}_{1}\right)=\operatorname{conv}\left(\left\{\mathrm{x}^{1}, \mathrm{x}^{2}, \mathrm{x}^{3}, \mathrm{x}^{4}, \mathbf{b}^{4}\right\}\right), \mathcal{P}_{2}=\Phi_{h}\left(\hat{\mathcal{P}}_{2}\right)=\operatorname{conv}\left(\left\{\mathrm{x}^{1}, \mathrm{x}^{2}, \mathbf{x}^{3}, \mathbf{b}^{3}, \mathbf{b}^{4}\right\}\right) \\
\mathcal{P}_{3}=\Phi_{h}\left(\hat{\mathcal{P}}_{3}\right)=\operatorname{conv}\left(\left\{\mathbf{x}^{1}, \mathbf{x}^{2}, \mathbf{b}^{2}, \mathbf{b}^{3}, \mathbf{b}^{4}\right\}\right), \mathcal{P}_{4}=\Phi_{h}\left(\hat{\mathcal{P}}_{4}\right)=\operatorname{conv}\left(\left\{\mathbf{x}^{1}, \mathbf{b}^{1}, \mathbf{b}^{2}, \mathbf{b}^{3}, \mathbf{b}^{4}\right\}\right) .
\end{gathered}
$$

Decomposition of the space-time interface into tetrahedra for case 1. The triangulation of the interface is trivially obtained with the tetrahedron

$$
\mathcal{P} \cap \mathcal{G}=\mathcal{I}=\operatorname{conv}\left(\left\{\mathbf{b}^{1}, \mathbf{b}^{2}, \mathbf{b}^{3}, \mathbf{b}^{4}\right\}\right) .
$$

5.4.2. Case 2: Decomposition into one 4-prism and one hypertriangle. Let us consider the case where two vertices of a pentatope are marked with a plus $(+)$, these are (w.l.o.g.) vertices $\mathbf{x}^{4}$ and $\mathbf{x}^{5}$. All other vertices $\left(\mathbf{x}^{1}, \mathbf{x}^{2}, \mathbf{x}^{3}\right)$ are marked with a minus (-). The cutting points of the hyperplane $\mathcal{G}$ with the edges are $\mathbf{c}^{1}:=\overline{\mathbf{x}^{1} \mathbf{x}^{4}} \cap \mathcal{G}$, $\mathbf{c}^{2}:=\overline{\mathbf{x}^{2} \mathbf{x}^{4}} \cap \mathcal{G}, \mathbf{c}^{3}:=\overline{\mathbf{x}^{3} \mathbf{x}^{4}} \cap \mathcal{G}, \mathbf{d}^{1}:=\overline{\mathbf{x}^{1} \mathbf{x}^{5}} \cap \mathcal{G}, \mathbf{d}^{2}:=\overline{\mathbf{x}^{2} \mathbf{x}^{5}} \cap \mathcal{G}, \mathbf{d}^{3}:=\overline{\mathbf{x}^{3} \mathbf{x}^{5}} \cap \mathcal{G}$. Thus we have to decompose the two parts $\mathcal{H}^{-}$and $\mathcal{Q}^{+}$into pentatopes with

$$
\begin{aligned}
\mathcal{H}^{-} & :=\operatorname{conv}\left(\left\{\mathbf{x}^{1}, \mathbf{x}^{2}, \mathbf{x}^{3}, \mathbf{c}^{1}, \mathbf{c}^{2}, \mathbf{c}^{3}, \mathbf{d}^{1}, \mathbf{d}^{2}, \mathbf{d}^{3}\right\}\right), \\
\mathcal{Q}^{+} & :=\operatorname{conv}\left(\left\{\mathbf{c}^{1}, \mathbf{c}^{2}, \mathbf{c}^{3}, \mathbf{d}^{1}, \mathbf{d}^{2}, \mathbf{d}^{3}, \mathbf{x}^{4}, \mathbf{x}^{5}\right\}\right) .
\end{aligned}
$$

Let us start with the decomposition of $\mathcal{H}^{-}$. Consider the mapping

$$
\Phi: \hat{\mathcal{H}} \rightarrow \mathcal{H}^{-},\left(\hat{x}_{1}, \hat{x}_{2}, \hat{x}_{3}, \hat{x}_{4}\right) \rightarrow \sum_{i=1}^{3} \sum_{j=1}^{3} \rho_{i}\left(\hat{x}_{1}, \hat{x}_{2}\right) \rho_{j}\left(\hat{x}_{3}, \hat{x}_{4}\right) \mathbf{q}^{i, j}
$$

with $\mathbf{q}^{i, 1}=\mathbf{x}^{i}, \mathbf{q}^{i, 2}=\mathbf{c}^{i}$ and $\mathbf{q}^{i, 3}=\mathbf{d}^{i}$ where $\rho_{i}\left(\hat{x}_{1}, \hat{x}_{2}\right)$ are the barycentric coordinates of the reference triangle $\hat{K} \subset \mathbb{R}^{2}$. Following section 5.3, we have a triangulation of $\hat{\mathcal{H}}$ into pentatopes $\left\{\hat{\mathcal{D}}_{i}\right\}$. One can show that the (pentatope-) piecewise linear interpolation $\Phi_{h}$ of $\Phi$ is an isomorphism between $\hat{\mathcal{H}}$ and $\mathcal{H}^{-}$and each image $\Phi_{h}\left(\hat{\mathcal{D}}_{i}\right)$ is again a pentatope. Therefore we can apply the decomposition of the reference 
hypertriangle $\hat{\mathcal{H}}$ into pentatopes to get the six pentatopes

$$
\begin{aligned}
\mathcal{D}_{u} & =\Phi_{h}\left(\hat{\mathcal{D}}_{u}\right)=\operatorname{conv}\left(\left\{\underline{\mathbf{x}}^{1}, \underline{\mathbf{x}}^{2}, \underline{\mathbf{x}}^{3}, \mathbf{c}^{2}, \mathbf{d}^{3}\right\}\right), \\
\mathcal{D}_{v} & =\Phi_{h}\left(\hat{\mathcal{D}}_{v}\right)=\mathbf{c o n v}\left(\left\{\mathbf{x}^{1}, \underline{\mathbf{c}}^{1}, \underline{\mathbf{c}}^{2}, \underline{\mathbf{c}}^{3}, \mathbf{d}^{3}\right\}\right), \\
\mathcal{D}_{w} & =\Phi_{h}\left(\hat{\mathcal{D}}_{w}\right)=\mathbf{c o n v}\left(\left\{\mathbf{x}^{1}, \mathbf{c}^{2}, \underline{\mathbf{d}}^{1}, \underline{\mathbf{d}}^{2}, \underline{\mathbf{d}}^{3}\right\}\right), \\
\mathcal{D}_{1}=\Phi_{h}\left(\hat{\mathcal{D}}_{1}\right) & =\mathbf{c o n v}\left(\left\{\underline{\mathbf{x}}^{1}, \underline{\mathbf{c}}^{1}, \mathbf{c}^{2}, \underline{\mathbf{d}}^{1}, \mathbf{d}^{3}\right\}\right), \\
\mathcal{D}_{2} & =\Phi_{h}\left(\hat{\mathcal{D}}_{2}\right)=\operatorname{conv}\left(\left\{\mathbf{x}^{1}, \underline{\mathbf{x}}^{2}, \underline{\mathbf{c}}^{2}, \underline{\mathbf{d}}^{2}, \mathbf{d}^{3}\right\}\right), \\
\mathcal{D}_{3}=\Phi_{h}\left(\hat{\mathcal{D}}_{3}\right) & =\operatorname{conv}\left(\left\{\mathbf{x}^{1}, \underline{\mathbf{x}}^{3}, \mathbf{c}^{2}, \underline{\mathbf{c}}^{3}, \underline{\mathbf{d}}^{3}\right\} .\right.
\end{aligned}
$$

We now turn over to $\mathcal{Q}^{+}$. For notational convenience define $\mathbf{c}^{4}:=\mathrm{x}^{4}$ and $\mathbf{d}^{4}:=\mathrm{x}^{5}$. Thus $\mathcal{Q}^{+}=\operatorname{conv}\left(\left\{\mathbf{c}^{1}, \mathbf{c}^{2}, \mathbf{c}^{3}, \mathbf{c}^{4}, \mathbf{d}^{1}, \mathbf{d}^{2}, \mathbf{d}^{3}, \mathbf{d}^{4}\right\}\right)$. Now the structure is similar to the situation for $\mathcal{Q}^{-}$in Case 1 and we can apply the same procedure and get a valid decomposition $\bigcup \mathcal{P}_{i}=\mathcal{Q}^{+}$with

$$
\begin{gathered}
\mathcal{P}_{1}=\Phi_{h}^{\mathcal{Q}}\left(\hat{\mathcal{P}}_{1}\right)=\operatorname{conv}\left(\left\{\mathbf{c}^{1}, \mathbf{c}^{2}, \mathbf{c}^{3}, \mathbf{x}^{4}, \mathbf{x}^{5}\right\}\right), \mathcal{P}_{2}=\Phi_{h}^{\mathcal{Q}}\left(\hat{\mathcal{P}}_{2}\right)=\operatorname{conv}\left(\left\{\mathbf{c}^{1}, \mathbf{c}^{2}, \mathbf{c}^{3}, \mathbf{d}^{3}, \mathbf{x}^{5}\right\}\right) \\
\mathcal{P}_{3}=\Phi_{h}^{\mathcal{Q}}\left(\hat{\mathcal{P}}_{3}\right)=\operatorname{conv}\left(\left\{\mathbf{c}^{1}, \mathbf{c}^{2}, \mathbf{d}^{2}, \mathbf{d}^{3}, \mathbf{x}^{5}\right\}\right), \mathcal{P}_{4}=\Phi_{h}^{\mathcal{Q}}\left(\hat{\mathcal{P}}_{4}\right)=\operatorname{conv}\left(\left\{\mathbf{c}^{1}, \mathbf{d}^{1}, \mathbf{d}^{2}, \mathbf{d}^{3}, \mathbf{x}^{5}\right\}\right)
\end{gathered}
$$

with $\Phi_{h}^{\mathcal{Q}}$ the corresponding piecewise linear transformation for the 4-prism.

Decomposition of the space-time interface into tetrahedra for case 2. With similar techniques as done for the four dimensional volume, we can proceed with the triangulation of the interface which is isomorph to a 3 -prism resulting in tetrahedra $\mathcal{I}_{i}$ :

$$
\mathcal{I}_{1}=\operatorname{conv}\left(\left\{\mathbf{c}^{1}, \mathbf{c}^{2}, \mathbf{c}^{3}, \mathbf{d}^{3}\right\}\right), \mathcal{I}_{2}=\operatorname{conv}\left(\left\{\mathbf{c}^{1}, \mathbf{c}^{2}, \mathbf{d}^{2}, \mathbf{d}^{3}\right\}\right), \mathcal{I}_{3}=\operatorname{conv}\left(\left\{\mathbf{c}^{1}, \mathbf{d}^{1}, \mathbf{d}^{2}, \mathbf{d}^{3}\right\}\right)
$$

Conclusion. The method presented and analysed in [20] is applied to a spatially three dimensional mass transport problem with a moving discontinuity. A strategy for constructing a piecewise planar approximate space-time interface and corresponding polygonal space-time domains is presented. For the integration on the polygonal space-time domains a method which decomposes the domain into a set of simplices is presented. Furthermore the construction of the tensor product space-time finite elements and the (non-standard) issue of quadrature rules in four space dimensions is addressed. Results of numerical experiments reveal third order in time convergence as long as the error related to the approximation of $\Gamma_{*}$ - which is of second order accuracy - is not dominating.

Acknowledgement. The author wants to thank Arnold Reusken for comments that greatly improved the manuscript. Financial support from the German Science Foundation (DFG) within the Priority Program (SPP) 1506 "Transport Processes at Fluidic Interfaces" is gratefully acknowledged.

Appendix A. Quadrature rule(s) on pentatopes. We shortly review lower order quadrature rules on pentatopes and discuss how to achieve higher order rules using tetrahedron rules, Duffy transformation and 1D Gauss-Jacobi integration rules. Integration rules are given for the reference pentatope $\hat{\mathcal{P}}$.

A.1. First order rule. There holds $\int_{\hat{\mathcal{P}}} 1 d \hat{\mathbf{x}}=1 / 24$ and $\int_{\hat{\mathcal{P}}} q(\hat{\mathbf{x}}) d \hat{\mathbf{x}}=1 / 120$ for $q(\hat{\mathbf{x}}) \in\left\{\hat{x}_{1}, \hat{x}_{2}, \hat{x}_{3}, \hat{x}_{4}\right\}$, s.t. the following rule is obviously exact for all polynomials up to degree one:

$$
I^{1}(f)=1 / 120 \sum_{i=0}^{4} f\left(\mathbf{e}^{i}\right)
$$



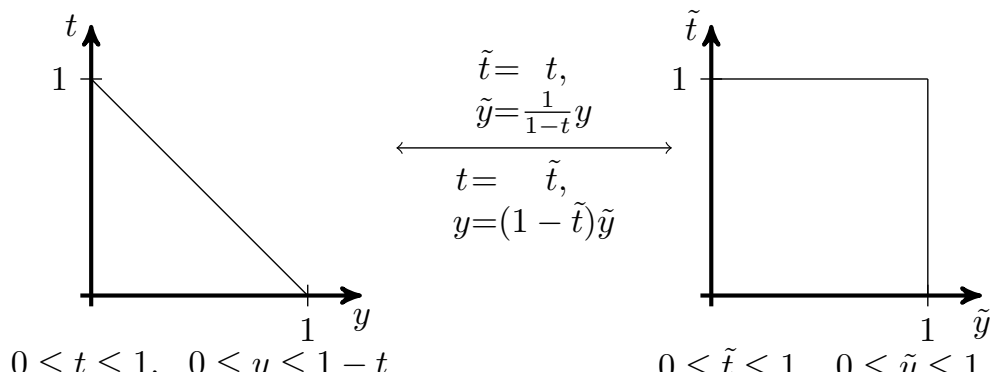

FIG. A.1. Sketch of the Duffy transformation for $d=1$

A.2. Third order rule. A third order rule, taken from [2], is as follows:

$$
I^{3}(f)=1 / 120 \sum_{i=1}^{5} f\left(\mathbf{s}^{i}\right) \text { with } \lambda_{j}\left(\mathbf{s}^{i}\right)=\alpha \text { for } i \neq j \text { and } \lambda_{j}\left(\mathbf{s}^{i}\right)=\beta \text { for } i=j
$$

with $\alpha=0.118350341907227374$ and $\beta=0.526598632371090503$.

A.3. Higher order rules using the Duffy transformation. A more general approach to derive integration rules for pentatopes is based on the Duffy transformation [7]. Let $\hat{\mathbf{y}}=\left(\hat{y}_{1}, \hat{y}_{2}, \hat{y}_{3}\right) \in \mathbb{R}^{3}$, and $\hat{\mathbf{x}}=(\hat{\mathbf{y}}, t) \in \mathbb{R}^{4}$. The problem to compute $\int_{\hat{\mathcal{P}}} f(\hat{\mathbf{x}}) d \hat{\mathbf{x}}=\int_{\hat{\mathcal{P}}} f(\hat{\mathbf{y}}, t) d(\hat{\mathbf{y}}, t)$ can be transformed using the transformation $(\hat{\mathbf{y}}, t) \rightarrow$ $(1 /(1-t) \hat{\mathbf{y}}, t)=(\tilde{\mathbf{y}}, t)$ (see also Figure A.1 for a sketch):

$$
\begin{aligned}
& \int_{\hat{\mathcal{P}}} f(\hat{\mathbf{y}}, t) d(\hat{\mathbf{y}}, t)=\int_{0}^{1} \int_{0}^{1-t} \int_{0}^{1-t-\hat{y}_{1}} \int_{0}^{1-t-\hat{y}_{1}-\hat{y}_{2}} f(\hat{\mathbf{y}}, t) d \hat{y}_{3} d \hat{y}_{2} d \hat{y}_{1} d t \\
& {[\tilde{\mathbf{y}}=1 /(1-t) \hat{\mathbf{y}}]} \\
& =\int_{0}^{1}(1-t)^{3} \int_{0}^{1} \int_{0}^{1-\tilde{y}_{1}} \int_{0}^{1-\tilde{y}_{1}-\tilde{y}_{2}} f((1-t) \tilde{\mathbf{y}}, t) d \tilde{y}_{3} d \tilde{y}_{2} d \tilde{y}_{1} d t \\
& =\int_{0}^{1}(1-t)^{3} \int_{\hat{T}} \tilde{f}(\tilde{\mathbf{y}}, t) d \tilde{\mathbf{y}} d t=\int_{0}^{1}(1-t)^{3} \tilde{g}(t) d t
\end{aligned}
$$

with $\tilde{f}(\tilde{\mathbf{y}}, t)=f((1-t) \tilde{\mathbf{y}}, t)$ and $\tilde{g}(t)=\int_{\hat{T}} \tilde{f}(\tilde{\mathbf{y}}, t) d \tilde{\mathbf{y}}$. In this form one can apply a one-dimensional integration rule of the form

$$
\int_{0}^{1}(1-t)^{3} \tilde{g}(t) d t \approx \sum_{k=0}^{N} \omega_{i} \tilde{g}\left(t_{i}\right)
$$

where $\omega_{i}$ and $t_{i}$ are weights and points of the corresponding quadrature rule. In order to approximate $\tilde{g}\left(t_{i}\right)$ at every integration point $t_{i}$ a standard 3D quadrature rule can be applied. Let's assume this 3D quadrature rule has order $q$ accuracy. The highest order for the pentatope rule at lowest costs is achieved if a Gauss-Jacobi rule (corresponding to the weight $(1-t)^{3}$ ) of order $q$ is used for the numerical integration w.r.t. $t$. The resulting quadrature rule is positive, but not symmetric. In principle also the quadrature rule for the tetrahedron can be derived from lower dimensional quadrature rules applying the idea recursively. This generic procedure generates quadrature rules which have slightly more points than symmetric Gauss rules. For that reason, we use symmetric Gauss rules for the tetrahedron. 
Appendix B. Computing the weighting factor $\nu$. The weighting factor $\nu$ in the Nitsche XFEM-DG method can be computed using the space-time normal $\mathbf{n}_{\Gamma_{*}}$ of the space-time interface. One can show that there holds

$$
\nu=\left(1+\left(\mathbf{w} \cdot \mathbf{n}_{\Gamma}\right)^{2}\right)^{-\frac{1}{2}}=\left\|\left(n_{1}, . ., n_{d}\right)^{T}\right\|
$$

with $\mathbf{n}_{\Gamma_{*}}=\left(n_{1}, . ., n_{d+1}\right)^{T}$ the space-time normal at the interface.

As we use a piecewise planar approximation of the space-time interface consisting of $\mathrm{d}$-simplices in $d+1$ dimensions we have to compute a normal to the $\mathrm{d}$-simplex. It is known that for $d=2$ one can use the standard cross-product to compute the normal. In the next section we quote a generalized cross-product which allows to do the same if $d=3$.

B.1. Computing normals to tetrahedra in 4 dimensions. In [14] a generalization of the cross-product is given. Given three vectors $\mathbf{u}^{1}, \mathbf{u}^{2}, \mathbf{u}^{3} \in \mathbb{R}^{4}$ one can compute the cross-product $\mathbf{v}=\mathbf{X}\left(\mathbf{u}^{1}, \mathbf{u}^{2}, \mathbf{u}^{3}\right)$, s.t.

- $\mathbf{X}\left(\mathbf{u}^{1}, \mathbf{u}^{2}, \mathbf{u}^{3}\right)=0$ iff $\mathbf{u}^{1}, \mathbf{u}^{2}, \mathbf{u}^{3}$ are linear dependent.

- Iff $\mathbf{u}^{1}, \mathbf{u}^{2}, \mathbf{u}^{3}$ are linear independent then for $\mathbf{v}=\mathbf{X}\left(\mathbf{u}^{1}, \mathbf{u}^{2}, \mathbf{u}^{3}\right)$, there holds: $\mathbf{v} \perp \mathbf{u}^{i}, i=1, . ., 3$.

- $\alpha \mathbf{X}\left(\mathbf{u}^{1}, \mathbf{u}^{2}, \mathbf{u}^{3}\right)=\mathbf{X}\left(\alpha \mathbf{u}^{1}, \mathbf{u}^{2}, \mathbf{u}^{3}\right)=\mathbf{X}\left(\mathbf{u}^{1}, \alpha \mathbf{u}^{2}, \mathbf{u}^{3}\right)=\mathbf{X}\left(\mathbf{u}^{1}, \mathbf{u}^{2}, \alpha \mathbf{u}^{3}\right), \alpha \in \mathbb{R}$

- $\mathbf{X}\left(\mathbf{u}^{1}, \mathbf{u}^{2}, \mathbf{u}^{3}\right)=\operatorname{sign}(\pi) \mathbf{X}\left(\mathbf{u}^{\pi(1)}, \mathbf{u}^{\pi(2)}, \mathbf{u}^{\pi(3)}\right)$, where $\pi$ is a permutation, i.e. changing the order of the arguments switches the sign.

This cross-product can be used to compute normals to tetrahedra. The computation is given below:

Given $\mathbf{u}, \mathbf{v}, \mathbf{w} \in \mathbb{R}^{4}$. Compute $\mathbf{z}=\mathbf{X}(\mathbf{u}, \mathbf{v}, \mathbf{w}) \in \mathbb{R}^{4}$ as follows:

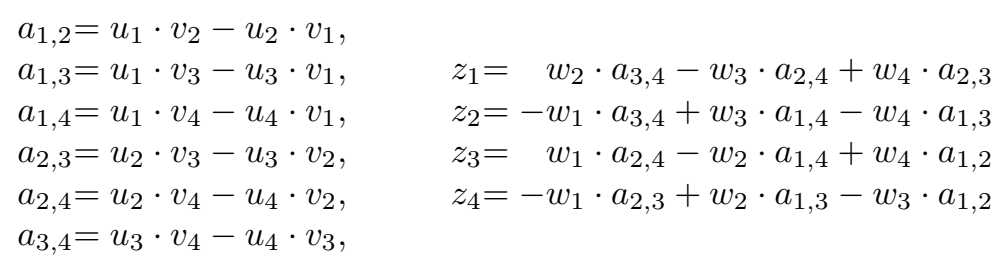

\section{REFERENCES}

[1] R. Becker, E. Burman, And P. Hansbo, A Nitsche extended finite element method for incompressible elasticity with discontinuous modulus of elasticity, Comput. Methods Appl. Mech. Engrg., 198 (2009), pp. 3352-3360.

[2] M. BeHR, Simplex space-time meshes in finite element simulations, Inter. J. Numer. Meth. Fluids, 57 (2008), pp. 1421-1434.

[3] D. Bothe, M. Koebe, K. Wielage, J. Prüss, and H.-J. Warnecke, Direct numerical simulation of mass transfer between rising gas bubbles and water, in Bubbly Flows: Analysis, Modelling and Calculation, M. Sommerfeld, ed., Heat and Mass Transfer, Springer, 2004.

[4] D. Bothe, M. Koebe, K. Wielage, And H.-J. Warnecke, VOF-simulations of mass transfer from single bubbles and bubble chains rising in aqueous solutions, in Proceedings 2003 ASME joint U.S.-European Fluids Eng. Conf., Honolulu, 2003, ASME. FEDSM2003-45155.

[5] A. Chernov and P. Hansbo, An hp-Nitsche's method for interface problems with nonconforming unstructured finite element meshes, Lect. Notes Comput. Sci. Eng., 76 (2011), pp. 153-161.

[6] J. Chessa and T. Belytschko, Arbitrary discontinuities in space-time finite elements by level sets and $x$-fem, Inter. J. Numer. Meth. Engng., 61 (2004), pp. 2595-2614.

[7] M. G. Duffy, Quadrature over a pyramid or cube of integrands with a singularity at a vertex, SIAM J. Numer. Anal., 19 (1982), pp. 1260-1262.

[8] S. Gross, V. Reichelt, And A. Reusken, A finite element based level set method for two-phase incompressible flows, Comp. Visual. Sci., 9 (2006), pp. 239-257.

[9] S. Gross and A. Reusken, Numerical Methods for Two-phase Incompressible Flows, Springer, Berlin, 2011. 
[10] A. Hansbo And P. Hansbo, An unfitted finite element method, based on Nitsche's method, for elliptic interface problems, Comput. Methods Appl. Mech. Engrg., 191 (2002), pp. 55375552 .

$[11] \longrightarrow$ A finite element method for the simulation of strong and weak discontinuities in solid mechanics, Comput. Methods Appl. Mech. Engrg., 193 (2004), pp. 3523-3540.

[12] A. Hansbo, P. Hansbo, and M. Larson, A finite element method on composite grids based on Nitsche's method, Math. Model. Numer. Anal., 37 (2003), pp. 495-514.

[13] P. Hansbo, C. Lovadina, I. Perugia, and G. Sangaldi, A Lagrange multiplier method for the finite element solution of elliptic interface problems using non-matching meshes., Numer. Math., 100 (2005), pp. 91-115.

[14] S. Holasch, Four-space visualization of 4 d objects, master's thesis, Arizona State University, 1991.

[15] B. Müller, F. Kummer, and M. Oberlack, Highly accurate surface and volume integration on implicit domains by means of moment-fitting, Int. J. Num. Meth. Eng., (2013).

[16] M. Muradoglu and G. Tryggvason, A front-tracking method for computation of interfacial flows with soluble surfactant, J. Comput. Phys., 227 (2008), pp. 2238-2262.

[17] M. Neumüller, Space-Time Methods, Fast Solvers and Applications, phd thesis, TU Graz, 2013.

[18] M. Neumüller and O. Steinbach, Refinement of flexible spacetime finite element meshes and discontinuous galerkin methods, Comp. Vis. Sci., 14 (2011), pp. 189-205.

[19] A. Reusken And C. Lehrenfeld, Nitsche-XFEM with streamline diffusion stabilization for a two-phase mass transport problem, SIAM J. Sci. Comp., 34 (2012), pp. 2740-2759.

[20] — Analysis of a Nitsche XFEM-DG discretization for a class of two-phase mass transport problems, SIAM J. Num. Anal., 51 (2013), pp. 958-983.

[21] A. Reusken and T. NGUYen, Nitsche's method for a transport problem in two-phase incompressible flows, J. Fourier Anal. Appl., 15 (2009), pp. 663-683.

[22] S. Sadhal, P. Ayyaswamy, and J. Chung, Transport Phenomena with Droplets and Bubbles, Springer, New York, 1997.

[23] J. Slattery, Advanced Transport Phenomena, Cambridge Universtiy Press, Cambridge, 1999.

[24] A. H. Stroud, Approximate calculation of multiple integrals, Math. Comput., 27 (1973), pp. $437-440$.

[25] V. Thomee, Galerkin finite element methods for parabolic problems, Springer, Berlin, 1997. 